\title{
THE APRIL MEETING IN BROOKLYN
}

The five hundred twelfth meeting of the American Mathematical Society was held on Thursday, Friday, and Saturday, April 14-16, 1955 , at the Polytechnic Institute of Brooklyn, which was celebrating its Centennial Year. A Symposium on Mathematical Probability and its Applications (sponsored by the Society and the Office of Ordnance Research) was held on Thursday and Friday. About 500 persons registered, including 392 members of the Society.

The Symposium was divided into three sessions which met at 10:00 A.M. and 2:00 P.M. on Thursday, and at 10:00 A.M. on Friday. The first session, devoted to Diffusion Theory, was presided over by Professor Shizuo Kakutani. The following papers were presented: Brownian motion depending on five parameters, by Professor Paul Lévy of l'École Polytechnique, Paris; $A$ new look at the Dirichlet problem, by Professor J. L. Doob of the University of Illinois; Probability and differential equations, by Professor William Feller of Princeton University.

At the second session of the Symposium, devoted to the Theory of Turbulence, the following papers were presented: Functional analysis in the theory of turbulence, by Professor Eberhard Hopf of Indiana University; Stochastic processes of interest in statistical astronomy, by Professor Guido Münch of the California Institute of Technology; The singularity in the spectrum of homogeneous turbulence, by Dr. G. K. Batchelor of Trinity College, Cambridge University. The chairman for this session was Professor Salomon Bochner.

Probability in Classical and Modern Physics was the subject of the third session at which Professor Herbert Robbins was the chairman. The papers presented were Probability in classical physics, by Professor Mark Kac of Cornell University; Infinite models in physics, by Dr. S. M. Ulam of Los Alamos, New Mexico; Quantum theory and the foundations of probability, by Professor B. O. Koopman of Columbia University.

By invitation of the Committee to Select Hour Speakers for Eastern Sectional Meetings, Professor R. D. Schafer of the University of Connecticut addressed the Society at 2:00 P.M. on Friday on Structure and representation of nonassociative algebras, and Professor Åke Pleijel of the University of Lund and the University of Maryland addressed the Society at 2:00 P.M. on Saturday on Eigenfunction distribution for polar differential equations. These sessions were presided over by Professors A. A. Albert and C. B. Morrey, Jr., respectively. 
Sessions for contributed papers were held at $3: 15$ P.M. on Friday, and at 10:00 A.M. and 3:15 P.M. on Saturday. Chairmen at these sessions were Professors J. B. Diaz, C. H. W. Sedgewick, Seymour Sherman, Wallace Givens, Drs. J. H. Curtiss, R. G. Bartle.

The ladies of the Department of Mathematics entertained at tea on Friday afternoon.

The Council of the Society met at 5:15 P.M. on Friday, reconvening after dinner.

The Secretary announced the election of the following thirty-nine persons to ordinary membership in the Society:

Mr. Eugene Albert, Brooklyn College;

Professor Norman Edward Albrecht, Hamline University;

Mr. Herbert Reeder Bailey, U. S. Naval Ordnance Plant, Indianapolis, Indiana;

Professor Herbert Barkan, Newark College of Engineering;

Mr. George Blustein, A. B. Dumont Laboratories, Passaic, New Jersey;

Mr. Jeffrey Jay Bowe, Air Force Cambridge Research Center, Cambridge, Massachusetts;

Mr. William Edward Boyce, Carnegie Institute of Technology;

Sister Theodora Breighner, O. S. U., Ursuline College, Louisville, Kentucky;

Mr. Fred C. Calabrese, Goodyear Aircraft Corporation, Akron, Ohio;

Mr. Joel Carroll, Argonne National Laboratory, Lemont, Illinois;

Miss Ellen Correl, Purdue University;

Mr. Cordell Evans, University of Pittsburgh;

Miss Catherine Mae Fitzpatrick, Rock Island Arsenal, Rock Island, Illinois;

Professor Masanori Fukaymiya, Tôhoku University, Sendai, Japan;

Mr. Herbert Zucker Hale, Astra Bent Wood Furniture Company, New York;

Miss Irene Harwick, Carnegie Institute of Technology;

Mr. Herbert Aaron Hauptman, Naval Research Laboratory, Washington, D. C.;

Mr. Michael Held, Institute of Mathematical Sciences, New York University;

Mr. Arnold Alfred Johnson, Goodyear Aircraft Corporation, Akron, Ohio;

Dr. Irving Kanter, Radio Corporation of America;

Mr. Raymond Kassler, Boland and Boyce, Inc., Belleville, New Jersey;

Mr. John David Leadley, University of Washington;

Mr. Robert Pierce Lee, Flight Determination Laboratory, White Sands Proving Ground;

Mr. Jacob Manheimer, Israeli Ministry of Defence, Tel-Aviv, Israel;

Mrs. Mary Kuskoff Neff, University of Florida;

Mr. Ernst Irvin Prasse, U. S. National Advisory Committee for Aeronautics, Cleveland, Ohio;

Mr. Leon Alexander Robbins, Park College, Parkville, Missouri;

Mr. William Henry Roudebush, U. S. National Advisory Committee for Aeronautics, Cleveland, Ohio;

Lt. Kenneth James Schlagheck, United States Air Force;

Professor Norman James Schoonmaker, University of Massachusetts;

Dr. John Robert Stock, National Carbon Research Laboratories, Cleveland, Ohio;

Miss Ellen Esther Swanson, American Mathematical Society, Providence, Rhode Island;

Mr. William Clement Swift, University of Kentucky; 
Mr. Robert Tates, Syracuse University;

Mr. Paul Haynes Thrower, University of Texas;

Sergeant Bruce Ramon Vogeli, Army Chemical Center, Maryland;

Mr. Alfred Weissberg, Battelle Memorial Institute;

Mr. Kenneth Franklin Wilson, University of Florida;

Mr. C. Rex Yeaman, Ricks College, Rexburg, Idaho.

It was reported that the following twenty-eight persons had been elected to membership on nomination of institutional members as indicated:

University of Chicago: Mr. Sterling Khazag Berberian, Dr. Wilfred Halliday Cockcroft, Mr. William L. Hoyt, Mr. Harold Irving Levine, Mr. Elon Lages Lima, Dr. Teruhisa Matsusaka, Dr. Jacob Pieter Murre, Mr. David Shale, Mr. Henry P. F. Swinnerton-Dyer, and Dr. Erik Christopher Zeeman.

Haverford College: Mr. W. Wistar Comfort.

Indiana University: Mr. Robert Edwin Heaton, Dr. Dietrich Morgenstern, Mr. Patrick Shanahan, Mr. Robert Clinton Wrede, and Mr. Arthur Jay Ziffer.

Institute for Advanced Study: Dr. Edoardo Storchi.

Michigan State College: Mr. William Leonard Harkness, Mr. Don Raymond Lick, and Mr. Frank Conant Sherburne, Jr.

New York University: Miss Betty Jane Gassner.

University of Tennessee: Mr. Ceslovas Masaitis.

University of Texas: Mr. Lewis Edwin Batson, Mr. Edwin Hale Connell, Mr. Victor A. Erma, Mr. Robert Heller, Jr., Mr. Richard Paul Kelisky, and Mr. William S. Mahavier.

The Secretary announced that the following had been admitted to the Society in accordance with reciprocity agreements with various mathematical organizations: Deutsche Mathematiker Vereinigung: Dr. Wolfgang Gaschutz, Universitate Kiel, Kiel, Germany, Professor Hermann Karl Schmidt, Universitat Wurzburg, Klinikstr., Germany, and Professor Egon Ullrich, Justus-Liebig University, Giessen, Germany; French Mathematical Society: Professor Maurice Audin, 22 rue de Nimes, Alger, Algerie, Mr. Pierre Brousse, 20 rue Boncenne, Pointers, Vienne, France, and Professor Sylvain Monavon, Lycée Parc Imperial, Nice, France; Indian Mathematical Society: Professor Balramji Rao Bhonsle, Government Engineering College, Jalapur, India; London Mathematical Society: Dr. Hanna Neumann, The University, Hull, England, and Mr. Jacob Jack Grannek, Queen's University, Belfast, Ireland; Swedish Mathematical Society: Professor Carl-Gustav Esseen, The Royal Institute of Technology, Stockholm, Sweden; Swiss Mathematical Society: Dr. Werner Goutschi, University of California, Berkeley; Wiskundig Genootschap Amsterdam: Mr. Maarten Jan Bossen, Technological University, Delft, Netherlands.

The following appointments by the President were reported: As a 
committee to nominate a representative of the Society on the Policy Committee for Mathematics: Samuel Eilenberg, Chairman, Marston Morse, and Antoni Zygmund; as a committee to nominate officers and members of the Council for 1956: H. F. Bohnenblust, Chairman, H. S. M. Coxeter, E. E. Moise, Deane Montgomery, and W. M. Whyburn; as a committee on arrangements for the 1955 Annual Meeting to be held at The Rice Institute: F. E. Ulrich, Chairman, H. E. Bray, A. H. Brown, L. K. Durst, H. M. Gehman, Guy Johnson, G. R. MacLane, S. Mandelbrojt, and J. W. T. Youngs; as a Committee to Select Gibbs Lecturers for 1956 and 1957: Marston Morse, Chairman, Garrett Birkhoff, and K. O. Friedrichs; as a committee on arrangements for the meeting to be held at the University of Tennessee, November 18-19, 1955: O. G. Harrold, Chairman, J. H. Roberts, W. S. Snyder, and R. L. Wilson; as representatives of the Society on the Organizing Committee for the second part of the Symposium on Mathematical Statistics and Probability to be held at the University of California at Berkeley during the summer of 1955: J. L. Doob and George Pòlya; as a joint committee of the Society and Association to investigate the possibility of holding the annual meeting at a time other than the week between Christmas and New Year's: R. M. Thrall, Chairman, H. M. Gehman, W. T. Martin, and J. M. Thomas; as a replacement for Professor T. R. Hollcroft on the Committee on the Employment Register: W. M. Hirsch.

The Secretary reported that the following persons have accepted invitations to deliver hour addresses during 1955: A. V. C. Pleijel, Brooklyn, New York, April 15-16; R. D. Schafer, Brooklyn, New York, April 15-16; A. H. Taub, Chicago, Illinois, April 22-23; Irving Kaplansky, Stanford, California, April 30; I. Barsotti, Vancouver, British Columbia, Canada, June 18; Raoul Bott, Ann Arbor, Michigan, Summer Meeting 1955; Edwin Hewitt, Ann Arbor, Michigan, Summer Meeting 1955; and Sze-Tsen Hu, Knoxville, Tennessee, November 18-19.

The following items were reported for the information of the Council: selection of W. T. Martin as Managing Editor of the Bulletin Editorial Committee for 1955; A. C. Schaeffer as Managing Editor of the Proceedings Editorial Committee for 1955; L. V. Ahlfors as Managing Editor of the Transactions and Memoirs Editorial Committee for 1955; Einar Hille as Chairman of the Mathematical Reviews Editorial Committee for 1955; Leo Zippin as Chairman of the Mathematical Surveys Editorial Committee for 1955; Salomon Bochner as Chairman of the Colloquium Editorial Committee for 1955 ; C. J. Rees as Chairman of the Committee on 
Printing and Publishing for 1955; and Dr. L. A. MacColl as Editor of the Proceedings of the Applied Mathematics Symposium held at Brooklyn Polytechnic Institute, April 14-15, 1955.

The following appointments to represent the Society were announced: Professor J. F. Randolph at the inauguration of Clifford Cook Furnas as Chancellor of the University of Buffalo on January 7 , 1955; Professor Wilfred Kaplan at the ceremonies commemorating the One Hundredth Anniversary of the founding of Michigan State College on February 12, 1955; Dr. Rufus Oldenburger at the inauguration of Leland Henry Carlson as President of Rockford College on February 27, 1955; Professor Paul A. White at the inauguration of Clark George Kuebler as Provost of Santa Barbara College on March 28, 1955.

The following actions taken by mail vote of the Council were reported: election of Professors Deane Montgomery and D. V. Widder to membership on the Executive Committee of the Council; election of the University of Alberta, Edmonton, Alberta, Canada, to institutional membership; approval of a recommendation of the Committee on Translations that the Translations Project be continued at the present level.

The Council voted to set meetings at Stanford University on April 30, 1955, and at the U. S. Naval Postgraduate School, Monterey, California, April 28, 1956, and voted to change the date of the October Meeting at the University of Maryland from October 29 to October 22, 1955.

The Council voted to approve the inclusion of the Society for Industrial and Applied Mathematics in the Combined List of Members.

The Council voted to discharge the Editorial Committee for Applied Mathematics Symposia Proceedings and to transfer the duties of this committee to the Applied Mathematics Committee.

The Council voted that the representatives of the Society on the Policy Committee for Mathematics shall be ex officio: the President, the President Elect or Ex-President as the case may be, the Secretary, and one of the Vice Presidents to be named each year by the President. The present representatives of the Society shall serve out their terms and the ex officio members shall be seated in the order named.

The Secretary reported the resignation of Professor J. W. Green as Associate Secretary for the Far West effective April 30, 1955. The Council elected Professor V. L. Klee as a replacement for Professor Green.

The Secretary reported that the Society for Industrial and Applied 
Mathematics had contributed $\$ 100$ toward the operation of the Employment Register. The Council voted to increase the membership of the Employment Register Committee from five to seven, two of the members to represent the Society for Industrial and Applied Mathematics.

The Council voted to approve an amendment to the by-laws increasing the membership of the Bulletin Editorial Committee from two to three.

The Council voted that it was the consensus of the Council that members of the Society should receive the Bulletin and Notices as a privilege of membership and that they should support the publication program of the Society to at least the extent they now do by subscribing for the Proceedings. The President was requested to appoint a committee to present a plan to implement this motion.

The Council approved a report from the Committee on Subsidies to Journals. Among the recommendations of this committee were the following: requests for new subsidies or for increases in present subsidies shall be addressed to the Secretary of the Society who will refer the request to the Committee on Printing and Publishing. This committee, after reviewing the request, will present its recommendation to the Council and Trustees for action; that subsidies to journals shall be computed on the basis of all papers published in the journal by individual members of the Society; that institutional dues are not to be considered as re-imbursement to the Society for publication costs but are to be based as closely as possible on the mathematical research activity on the campus of the institution.

The Council voted to write to the Senate Judiciary Committee in support of a personal bill which would exempt Professor Hermann Weyl from a provision of the McCarran-Walter Act which might cause the loss of his American citizenship.

Abstracts of the contributed papers follow. Those with " $t$ " after the abstract number were presented by title. In the case of joint papers, the name of the author who read the paper is followed by (p). Dr. Stoll was introduced by Professor W. H. Gottschalk, Dr. Reiner by Professor Irving Reiner, and Dr. Kreyszig by Professor Stefan Bergman.

\section{Algebra and Theory of Numbers}

441t. Armand Borel and G. D, Mostow: On semi-simple automorphisms of Lie algebras.

This paper investigates the existence of fixed points and invariant Cartan subalgebras under automorphisms of (finite-dimensional) Lie algebras over an arbitrary 
field of characteristic 0 . It is shown that a semi-simple (i.e. with simple elementary divisors) automorphism of a nonsolvable Lie algebra $L$ leaves fixed an element $x$ with non-nilpotent ad $x$, and which is regular if $L$ is semi-simple. As to the second question, the main result is: Let $G$ be a group of semi-simple automorphisms of a Lie algebra $L$. Then $G$ leaves a Cartan subalgebra invariant when $G$ and $L$ are solvable or when $L$ is arbitrary and $G$ has type (MP)*. Here, a linear group $G$ is said to be of type (MP)* if it contains an increasing sequence of invariant subgroups $\left(G_{i}\right)$ $\left((0 \leqq i \leqq s), G_{0}=\{e\}, G_{s}=G\right)$, such that $G_{i+1}$ is generated by $G_{i}$ and by an abelian subgroup $H_{i}$ which either is cyclic or has a connected algebraic hull $(0 \leqq i<s)$. This result generalizes a theorem of J-P. Serre and one of the authors (Comment Math. Helv. vol. 27 (1953) pp. 128-139). It is also shown that an automorphism of a semisimple Lie algebra leaving a Cartan subalgebra invariant must be semi-simple. (Received February 7,1955 .)

442. Abraham Brind: The summation which forms the basis for proving both Fermat theorems.

The author proves by mathematical induction: If $y$ and $n$ are two arbitrary positive integers, then $y^{n}= \pm y+\sum_{r=1}^{r=y} \sum_{s=1}^{s=n-1}(-1)^{s-1} C_{n, s} r^{n-s}$, where the plus sign must be taken when $n$ is odd and the minus sign when $n$ is even. He then derives two important particular cases, one for $n=p$, any odd prime, and the other for $n=2$, the only even prime. In the latter case he establishes another summation distinct from the first both in the matter of the limits of summation and in the sign of $y$. The application of the above to the so-called Fermat little theorem is shown to be immediate. But the author can only allude to the somewhat lengthy proof of Fermat's last theorem which he hopes to have ready for publication in the near future. The infinite descent method is not used at all. Nor is unique decomposition used in the proof of the odd (prime) case. A more basic property of integers is used to distinguish them from irrationals (the necessity of using complex numbers and fractional rational numbers is easily eliminated): the inductive property of positive integers. The proof is effected by comparing a summation derived from the Fermat equation with the above. Unique decomposition is used in the complete solution of the Pythagorean case (in which, by the way, a very significant analytical meaning of some expressions is pointed out) and in the proof of the impossibility of the equation when $n=4$. (Received March 1, 1955.)

\section{3t. Leonard Carlitz: Note on the class number of real quadratic} fields.

Let $d$ denote the discriminant and $h(d)$ the class number of the real quadratic field $R\left(d^{1 / 2}\right)$; also let $\epsilon=\left(t+u d^{1 / 2}\right) / 2$ denote the fundamental unit of the field, $\epsilon>1$. For $d=p, p$ prime $>2$, Ankeny, Artin, and Chowla (Proc. Nat. Acad. Sci. U.S.A. vol. 37 (1951) pp. 524-527) have stated the formula $2 u h(p) / t \equiv(A+B) / p(\bmod p)$, where $A$ is the product of the quadratic residues of $p$ and $B$ is the product of the nonresidues in the interval $1, p-1$. In the present paper the following more general result is proved. Let $d=p m>0, p>m>1$ and put $A=\prod_{1}^{d} a^{\left(m_{0} / a\right)}, B=\prod_{1}^{d} b^{\left(m_{0} / b\right)}$, where in the first product $(a / p)=+1$, while in the second $(b / p)=-1$, and $m_{0}=(-1)^{(p-1) / 2} m$. Then $2 m u h(d) / t \equiv(B-A) / p(\bmod p)$. (Received February $25,1955$.

444t. Leonard Carlitz: On the representation of an integer as the sum of twenty-four squares. 
Making use of the methods developed in earlier papers, van der Pol (Proc. Neder. Akad. Wetensch. Ser. A57 = Indagationes Math. vol. 16 (1954) pp. 349-361) has given an elegant proof of Ramanujan's formula for the number of representations of an integer as the sum of twenty-four squares. The present paper contains a short proof of the formula which assumes only certain standard results from the theory of elliptic functions. (Received February 25, 1955.)

445t. Leonard Carlitz and J. H. Hodges: Distribution of matrices in a finite field with respect to characteristic polynomial and invariant factors.

In this paper the distribution of square matrices, with elements in $G F(q)$, having certain properties is studied by examining the conditions imposed by these properties on the characteristic polynomials of the matrices. The number of nonderogatory matrices of given order is found in terms of the number of nonsingular matrices of that order and the Euler function for $G F[q, x]$, the polynomial ring in $x$ over $G F(q)$. Matrices $A$ and $B$ are said to be in the same class if and only if $A$ is similar to $B$. The characteristic polynomial of a matrix $A$ of order $m$ is $F(\lambda)=H_{1}(\lambda) H_{2}(\lambda) \cdots H_{m}(\lambda)$, where $H_{i+1} \mid H_{i}$ and the $H_{i}$ are the invariant factors of $\lambda I-A$. The number of classes of order $m$ having a given $F(\lambda)$ as characteristic polynomial, the total number of classes of order $m$, and the number of classes of order $m$ having $H_{1}(\lambda)$ of degree $r$ are all found in terms of certain types of partitions of positive integers. (Received February $25,1955$.

\section{6t. Eckford Cohen: $A$ class of arithmetical functions.}

Let $r$ be an integer $>1$ and suppose $f(n)$ to be an arithmetical function of period $r$. Further place $(n, r)=h$; then $f(n)$ is defined to be an even function $(\bmod r)$ provided $f(n)=f(h)$ for all $n$. The following theorem is proved: An arithmetical function $f(n)$ of period $r$ is even if and only if it is expressible in the form $\sum_{d \mid r} \alpha(d) c(n, d)$ where $c(n, d)$ is Ramanujan's sum. The proof of this result is based on an orthogonality property of $c(n, d)$ due to the author (Duke Math. J. vol. 19 (1952) p. 120, (3.10)). It is also shown that the even functions $(\bmod r)$ include the class of functions introduced by Anderson and Apostol (Duke Math. J. vol. 20 (1953) pp. 211-216). Using the above theorem, inversion formulas for even functions are obtained. In special cases these formulas reduce to results of Nicol and Vandiver (Proc. Nat. Acad. Sci. U.S.A. vol. 40 (1954) pp. 825-835, Theorems V and VI). A new proof of Theorem VII of Nicol and Vandiver on restricted partitions $(\bmod r)$ is also given. (Received March 31, 1955.)

\section{7t. Karl Goldberg: Bounds and asymptotic behavior of coefficients in the series $\log e^{x} e^{y}$.}

In the formal power series for $\log e^{x} e^{y}$ when $x$ and $y$ do not commute let $c_{x}\left(s_{1}, \cdots\right.$, $s_{m}$ ) denote the coefficient of the term $x^{s_{1}} y^{s_{2}} \cdots(x, y)^{s_{m}}$ with $s_{i} \geqq 1$ for all $i$. The author proves $\left|c_{x}\left(s_{1}, \cdots, s_{m}\right)\right| \leqq 1 / 2^{d} M$ with equality when $d$ vanishes where $d=\sum_{i=1}^{m}\left(s_{i}-1\right)$ and $M=m ! /[m / 2] ![(m-1) / 2] !$ with $[a]$ denoting the largest integer in $a$. It is conjectured that the constant 2 in the inequality can be replaced by the constant $\pi$. Furthermore let $C(m, d)=\max \left|c_{x}\left(s_{1}, \cdots, s_{m}\right)\right|$ where the $s_{i}$ range over all values such that $\sum_{i=1}^{m}\left(s_{i}-1\right)=d$. The author proves that for a fixed $d: \lim _{m \rightarrow \infty} M C(m, d)$ $=4\left(2^{d+2}-1\right)\left|B_{d+2}\right| /(d+2) !$ if $d$ is even and $\lim _{m \rightarrow \infty} m M C(m, d)=8(d+2)\left(2^{d+8}-1\right)$ 
- $\left|B_{d+3}\right| /(d+3)$ ! if $d$ is odd where $B_{i}$ is the $i$ th Bernoulli number in the usual notation $\left(B_{1}=-1 / 2\right)$. These theorems also hold for the coefficients $c_{y}\left(s_{1}, \cdots, s_{m}\right)$ of terms starting with a positive power of $y$ since $\left|c_{x}\left(s_{1}, \cdots, s_{m}\right)\right|=\left|c_{y}\left(s_{1}, \cdots, s_{m}\right)\right|$. (Received February 17, 1955.)

\section{Emilie V. Haynsworth: Characteristic roots of certain matrices. Preliminary report.}

As one result of a recent paper by the author (Quasi-stochastic matrices, to be published), it was shown that any $n \times n$ matrix $A=\left(a_{i j}\right)$, with $\sum_{j=1}^{k} a_{i j}=r_{11}, \sum_{j=k+1}^{n} a_{i j}$ $=r_{12}(i=1, \cdots, k), \sum_{j=1}^{k} a_{i j}=r_{21}$, and $\sum_{i=k+1}^{n} a_{i j}=r_{22} \quad(i=k+1, \cdots, n)$, has two roots which are roots of the matrix $R=\left(r_{i j}\right)(i, j=1,2)$. In this paper the result is generalized to matrices which can be partitioned into $m^{2}$ submatrices, $m<n$, with row-sums, $r_{i j}(i, j=1, \cdots, m)$. Such matrices have as $m$ of their roots the roots of the matrix $R=\left(r_{i 4}\right)$. It is also shown that the other $n-m$ roots of $A$ are roots of an $(n-m)^{2}$ matrix whose elements can be found by inspection from the elements of $A$. These results are proved by using theorems from a paper by L. S. Goddard ( $A n$ extension of a matrix theorem by A. Brauer, not yet published). (Received January $14,1955$.

\section{K. A. Hirsch: On locally nilpotent groups.}

On another occasion (International Congress of Mathematicians, Cambridge, 1950; Mathematische Nachrichten vol. 3 (1951)) the author has proved: Theorem 1: A group with maximal condition for subgroups in which every proper subgroup differs from its normaliser is nilpotent. It is plausible to ask whether the maximal condition can be weakened or omitted altogether, provided the conclusion is weakened correspondingly. In this direction B. I. Plotkin (Doklady Akad. Nauk USSR vol. 76 (1951); see also Kuroš, Teoriya Grupp, 2d ed., Moscow, 1953, §63) has proved: Theorem 2: A group in which every proper subgroup differs from its normaliser is locally nilpotent. It will now be shown that a stronger form of the arguments that have been used in the proof of Theorem 1 is sufficient to give a simple proof of Theorem 2. For this purpose a theorem that can claim interest on its own account is required. Theorem 3: Every group $G$ possesses a unique maximal locally nilpotent normal subgroup that contains all locally nilpotent normal subgroups of $G$. Theorem 3 is a consequence of: Theorem 4: The union of two locally nilpotent normal subgroups of an arbitrary group $G$ is itself a locally nilpotent normal subgroup of $G$. A full account will appear elsewhere (Schur memorial volume of Math. Zeit.). (Received February 25, 1955.)

\section{0t. J. H. Hodges: Explicit values and applications of certain exponential sums in a finite field.}

In Representations by quadratic forms in a finite field (Duke Math. J. vol. 21 (1954) pp. 123-138) and Representations by skew forms in a finite field (Archiv der Mathematik vol. 5 (1954) pp. 19-31), L. Carlitz has defined certain exponential sums for symmetric and skew matrices, respectively, over a finite field. The present paper is concerned with finding the explicit values of these sums and with applications of the sums to the solution of a number of matric problems. For example, the number of ways to partition a given symmetric matrix $A$ of order $t$ into the sum of $n(n>1)$ symmetric matrices of order $t$ having assigned ranks and invariants is found. (Received February 28, 1955.) 
451t. M. D. Marcus: An eigenvalue inequality for the product of normal matrices.

If $A$ and $B$ are $n$-square normal matrices with eigenvalues $\lambda_{i}(A)$ and $\lambda_{i}(B)$, $i=1, \cdots, n$, then it is proved that $\min _{p} \operatorname{Re} \sum_{i=1}^{n} \lambda_{i}(A) \lambda_{p(i)}(B) \leqq \operatorname{Re} \sum_{i=1}^{n} \lambda_{i}(A B)$ $\leqq \max _{p} \operatorname{Re} \sum_{i=1}^{n} \lambda_{i}(A) \lambda_{p(i)}(B)$ where $p$ runs over all permutations of the integers 1 , $2, \cdots, n$. (Received February 21, 1955.)

\section{2t. M. D. Marcus: $A$ note on matrix inequalities.}

Let $\lambda(A), \lambda_{m}(A)$, and $\lambda_{M}(A)$ denote an arbitrary eigenvalue of $A$, the minimum, and the maximum eigenvalues of $A$ respectively where $A$ is an arbitrary $n$-square matrix. Let $\|x\|^{2}=\sum_{i=1}^{n}\left|x_{i}\right|^{2}$. Results: (i) If $C$ and $D$ are arbitrary, $|\lambda(C D)|^{2}$ $\leqq \lambda_{M}\left(C C^{*}\right) \lambda_{M}\left(D D^{*}\right)$. If there exist $z_{0}$ and $z_{1}$, unit vectors minimizing $\left\|C^{*} C D D^{*} z\right\|$ and $\left\|D D^{*} C^{*} C z\right\|$ respectively, such that $\left\|D D^{*} z_{0}\right\| \neq 0$ or $\left\|C^{*} C z_{1}\right\| \neq 0$ then $|\lambda(C D)|^{2}$ $\geqq \lambda_{m}\left(C C^{*}\right) \lambda_{m}\left(D D^{*}\right)$. (Compare S. N. Roy, Proc. Amer. Math. Soc. vol. 5 (1954) pp. 635-638.) (ii) Let $C=A+i B$ be Hermitian and $i_{1} \leqq i_{2} \leqq \cdots \leqq i_{k}, k \leqq n$ be integers. Then $k \lambda_{m}(C) \leqq \sum_{a, \beta_{m=1}}^{k} A_{i_{\alpha} i_{\beta}} \leqq k \lambda_{M}(C)$. (iii) If $C$ is arbitrary then it is proved that $k \lambda_{m}\left(C C^{*}\right) \leqq \sum_{a, \beta=1}^{k} \sum_{j=1}^{n} C_{i_{\alpha} j} \bar{C}_{i \beta^{j}} \leqq k \lambda_{M}\left(C C^{*}\right)$, extending a result of W. V. Parker (Duke Math. J. vol. 15 (1948) pp. 711-715). (Received February 21, 1955.)

\section{3t. G. D. Mostow: Fully reducible subgroups of algebraic groups.}

Let $G$ be an algebraic matric group over an arbitrary field of characteristic zero. Let $G$ denote its Lie algebra and $\mathcal{N}$ the maximum ideal of nilpotent matrices. Denote by $N$ the corresponding connected algebraic subgroup of $G$. Theorem. If $M$ is any maximal fully reducible subgroup of $G$, then $G=M \cdot N$ semi-directly. The maximal fully reducible subgroups of $G$ are conjugate under an inner automorphism. Theorem. Let $E$ be the associative enveloping algebra of $G$. There is a Wedderburn decomposition $S+T$ for $E$ with (a) $S \cap G$ maximal fully reducible in $G$; (b) $(I+T) \cap G=N$, $I=$ identity; and (c) $T \cap G=\mathcal{N}$. Theorem. A fully reducible group of automorphisms of a Lie (resp. associative or Jordan) algebra $G$ keeps invariant a maximal semi-simple subalgebra; it keeps invariant a Cartan subalgebra if $G$ is solvable. An intermediate result is: Theorem. A rational representation carries fully reducible groups into fully reducible groups. A corollary of this is: The first main theorem of the theory of invariants holds for fully reducible groups. (Received March 1, 1955.)

\section{4t. G. D. Mostow: Self-adjoint groups.}

Theorem. Let $G_{1}, G_{2}, \cdots, G_{n}$ be a nested sequence of fully reducible algebraic groups of automorphisms of a real linear space $V$. Then a positive definite inner product can be introduced on $V$ with respect to which $G_{1}, G_{2}, \cdots, G_{n}$ are self-adjoint. The proof utilizes the notion of "invariant compact form" which is defined for groups which are not necessarily connected. The basic steps in the proof are: (1) A fully reducible real algebraic group has an invariant compact form; (2) If $F \subset G$ and each has an invariant compact form, then any invariant compact form for $F$ can be extended to an invariant compact form for $G$. Among the intermediate results: Theorem. A Lie group with a finite number of connected components is topologically the direct product of a compact subgroup $K$ and a euclidean subspace $E$ with $k E k^{-1}=E$ for $k$ in $K$. All maximal compact subgroups are conjugate under inner automorphisms. A corollary of the main theorem for the case $n=1$ is: If $H$ is a closed connected sub- 
group of a Lie group $G$ with (Ad $H)_{G / R}$ fully reducible, then $G / H$ admits a covariant fibering ( $R$ denoting the radical). (Received March 1, 1955.)

\section{5t. Morris Newman and Olga Taussky: Unimodular circulants of rational integers.}

It is shown that there are nontrivial unimodular $n \times n$ circulants with rational integers as elements for all $n$ except 2, 3, 4, 6 (a circulant is called trivial if it contains only one nonzero element in each row). If $n$ is a power of a prime and such a circulant is of the form $A A^{\prime}$ ( $A^{\prime}$ the transpose of $A$ ) where $A$ is an $n \times n$ matrix with rational integers as elements, then $A$ is itself the product of a circulant, a permutation matrix, and a diagonal matrix consisting of \pm 1 's. These results are applied in a study of the normal basis of algebraic number fields. (Received February 10,1955.)

\section{6t. Irma M. Reiner: On the two-adic density of representations by quadratic forms.}

The problem of determining $A_{q}(S, T)$, the number of solutions of $X^{\prime} S X \equiv T$ $(\bmod q)$, where $S$ and $T$ are symmetric integral matrices, has been considered by C. L. Siegel (Ann. of Math. vol. 36 (1935) pp. 527-606). He obtained explicit formulas for $A_{q}(S, T)$ when $q=p^{a}$, where $p$ is a prime not dividing $2|S||T|$. He further showed that in the case where $|S||T|$ is odd, the determination of $A_{r}(S, T)$ for $r=2^{b}, b \geqq 3$, reduces immediately to that of finding $A_{8}(S, T)$. In this paper formulas are found for $A_{2}(S, T)$ and $A_{8}(S, T)$, assuming that $|S||T|$ is odd. A combinatorial argument is used when $q=2$, and exponential sums are employed when $q=8$. The proof uses 2-adic canonical forms of B. W. Jones and Gordon Pall (Duke Math. J. vol. 11 (1944) pp. 715-727). (Received February 21, 1955.)

\section{7t. E. V. Schenkman: Groups with Abelian derived groups.}

The author constructs a universal semigroup $S$ and gives its multiplication table explicitly so that if $G$ is a group generated by elements of finite order and if $G$ has Abelian commutator subgroup, then $G$ is a homomorphic image of $S$. In particular he gives a presentation and multiplication table for the free product of finite cyclic groups modulo the second derived group of the free product. (Received February 24, 1955.)

\section{8t. Ernst Snapper: Higher-dimensional field theory. I. The integral closure of a module.}

Let $E / F$ be a finitely generated field extension, i.e., $E$ and $F$ are commutative fields and $E=F\left(a_{1}, \cdots, a_{n}\right)$, where $a_{1}, \cdots, a_{n}$ belong to $E$. The present paper is the first of a series of three articles which have as purpose to develop an intrinsic theory for $E / F$, by giving a purely field-theoretic treatment of the theory of linear systems of algebraic varieties. Let $L$ denote a finitely generated module of $E / F$. See the author's paper Integral closure of modules and complete linear systems, Bull. Amer. Math. Soc. Abstract 61-3-399, for the notion of the integral closure $|L|_{i}$ of $L$ and the fact that $|L|_{i}$ is finitely generated. In the present paper, the theory of $|L|_{i}$ is further developed. In particular, it is proved that there exists an integer $k_{0}$ such that, if $k \geqq k_{0}$, $\left|L^{k+s}\right|_{i}=\left|L^{k}\right|_{i} L^{s}$ for all $s \geqq 0$. This fact constitutes the main theorem concerning integral closures of modules and will enable us in the subsequent articles to give a purely field-theoretic treatment of Zariski's theory of normalization. (Received March 4, 1955.) 


\section{ANALYSIS}

459. Joseph Andrushkiw: On the minimum maximorum and maximum minimorum of a polynomial whose all zeros are real.

Let $z_{1}, z_{2}, \cdots, z_{n}$ denote the zeros of the polynomial $f(z)=1+a_{1} z+\cdots+a_{n} z^{n}$, $z_{1}^{*}, z_{2}^{*}, \cdots, z_{n}^{*}$ the zeros of the polynomial $f(z ; x)=f(z)-x, s_{-k}=\sum_{j-1}^{n} z_{j}^{-k}, s_{-k}^{*}$ $=\sum_{j=1}^{n} z_{i}^{*-k}, D_{k}$ and $D_{k}(x)$ the determinants $\left|s_{-(i+j)}\right|,\left|s_{-(i+j)}^{*}\right|, i, j=1,2, \cdots, k$; $k=1,2, \cdots, n$, respectively. $D_{n}(x)$ expressed in terms of coefficients of $f(z ; x)$ is a polynomial of $n-1$ st degree in $x$ and equals the discriminant of $f(z ; x)$ multiplied by a factor (author's paper, Bull. Amer. Math. Soc. Abstract 58-3-289). It follows that the zeros of $D_{n}(x)$ are $f\left(\alpha_{k}\right)$ where $\alpha_{k}$ are the zeros of the derivative $f^{\prime}(z)$. If all zeros of $f(z)$ are real and distinct, the greatest negative zero $N$ of $D_{n}(x)$ represents maximum minimorum of $f(z)$ and the smallest positive $P$ minimum maximorum. Since the zeros of a polynomial are continuous functions of its coefficients, it follows that $x_{1}<N<P$ $<x_{2}$ where $x_{1}$ and $x_{2}$ are zeros of the quadratic equation $D_{2}(x)=0$. Cauchy's (M. Marden: The geometry of zeros of a polynomial, p. 98, ex. 2) and Hadamard's theorems together with the above result yield the inequality: $-\left(c+\left(c^{2}+4 a b\right)^{1 / 2}\right) / 2 b<N<-D_{n}$ - $\left[D_{n}+C(n, p)\left(n^{2}+n\right)^{n / 2} M^{2 n-1}\right]^{-1}<0<D_{n}\left[D_{n}+C(n, p)\left(n^{2}+n\right)^{n / 2} M^{2 n-1}\right]^{-1}<P<(-c$ $\left.+\left(c^{2}+4 a b\right)^{1 / 2}\right) / 2 b$, where $a>0, b>0$, and $c$ are assumed to be polynomials in $a_{1}, a_{2}, a_{3}$, $a_{4}, p=[(n+1) / 2]$ and $M=\max \left(1, k a_{k}\right), k=1,2, \cdots, n$. (Received February 7,1955 .)

\section{0t. Stefan Bergman: Bounds for the stream function of a com-} pressible fluid.

The author considers the stream function $\psi(\mathrm{H}, \theta)$ of compressible fluids in the $\mathbf{H}, \theta$-plane. (Here $\mathbf{H}$ is a conveniently chosen function of the Mach number and $\theta$ the angle which the velocity vector forms with the positive $x$-direction. (See Amer. J. Math. vol. 70, p. 859 ff., (2.1), (2.2).) $\psi$ satisfies the equation $l(\mathrm{H}) \psi_{\theta \theta}+\psi_{\mathrm{HH}}=0$, where $l(\mathbf{H})>0, \mathbf{H}<0$ in the subsonic region, $l(\mathbf{H})<0, \mathbf{H}>0$ in the supersonic region. The author assumes that $a_{1}^{2} \mathrm{H}^{P}<|l(\mathrm{H})|<a_{2}^{2} \mathrm{H}^{P}$, where $0<a_{1}<a_{2}<\infty, P$ is an integer, and, for $\mathrm{H}<0, P$ is odd. If $\psi(0, \theta)=\sum_{n=0}^{p} C_{n} \theta^{n}, \psi_{\mathrm{H}}(\mathrm{H}, \theta)_{\mathrm{H}-0}=0, \psi_{\mathrm{H}} \equiv \partial \psi / \partial \mathrm{H}$, then $\psi(\mathrm{H}, \theta)$ $\leqq \sum_{n=0}^{P} \quad \sum_{\mu_{n=1}}^{2}(-1)^{\mu}\left((-1)^{\mu} C_{n} \theta^{n}\right)^{+} F\left[-n / 2,(-n+1) / 2,(P+1) /(P+2) ; 4 a_{\mu}^{2}|\mathbf{H}|^{P+2}\right.$ $\left.\cdot(P+2)^{-2} \theta^{-2}\right]$ where $F$ is the hypergeometric function; $a^{+}=a$ if $a \geqq 0, a^{+}=0$ if $a<0$. Replacing $(-1)^{\mu}$ by $(-1)^{\mu+1}$ yields a lower bound. If $\psi(0, \theta)=0, \psi_{\mathrm{H}}(\mathrm{H}, \theta)_{\mathrm{H}-0}$ $=\sum_{n=1}^{P} D_{n} \theta^{n}$, then it is proved under analogous assumptions for $\mathrm{H}>0$ that $\psi_{\theta}(\mathrm{H}, \theta)$ $\leqq \sum_{n=1}^{n \rightarrow 1} \sum_{\mu_{n=1}}^{2}(-1)^{\mu+1}\left((-1)^{\mu+1} n D_{n} \theta^{n-1} \mathrm{H}\right)^{+} F[(-n+1) / 2,-n / 2+1,(P+3) /(P+2)$; $\left.4 a_{3-\mu} \mathrm{H}^{P+2}(P+2)^{-2} \theta^{-2}\right]$. A similar formula holds for a lower bound, as well as for $\mathbf{H}<0$. (Received April 11, 1955.)

461t. H. S. Collins: An extension theorem for completely regular spaces.

Let $E$ be a completely regular $T_{1}$ topological space, $A$ and $M$ closed subsets, and denote by $C(E)$ [by $C_{E}(M)$ ] the set of real continuous functions on $E$ [the set of real continuous functions on $M$, each of which has a continuous extension over $E$ ]. In what follows, statements involving a uniformity refer to the weakest uniform structure on $E$ for which all $f \in C(E)$ are uniformly continuous. Theorem. If $A$ is totally bounded and $f$ real continuous on $M$ is such that $|f(t)|<1$, all $t \in A \cap M$, the following two conditions together are sufficient that there be $g \in C(E)$, an extension of $f$, such that $|g(t)|<1$, all $t \in A$ : (1) $f \in C_{E}(M)$, and (2) either $A$ or $M$ is complete. If $\mathfrak{A}$ is a nonvoid family of subsets of $E$, endow $C(E)\left[C_{E}(M)\right]$ with the topology of uniform con- 
vergence on the members of $\mathfrak{A}$ [of $\{A \cap M: A \in \mathfrak{A}\}$ ]. Define $T$, for $f \in C(E)$, by $T(f)=f$ restricted to $M$. Corollary. $T$ is open onto $C_{E}(M)$ if either (1) $\mathfrak{A}=$ all compact sets, or (2) $\mathfrak{A}=$ all totally bounded sets and $M$ is complete or each closed $A \in \mathfrak{P}$ is complete. (Received February 18, 1955.)

\section{2t. Albert Edrei: On the zeros of successive derivatives.}

Consider an analytic function $f(z)$, regular at $z_{0}$, and assume (i) that if $f(z)$ is entire, it is not of exponential type; (ii) that $z_{0}$ is not a limit point of the zeros of the successive derivatives of $f(z)$. Then, either the sequence (1) $\left\{\left|f^{(n+1)}\left(z_{0}\right) / f^{(n)}\left(z_{0}\right)\right|\right\}$ tends to infinity or else, for all sufficiently large values of the positive number $P$, every interval $[P, P Q]$ contains a limit point of (1). The positive quantity $Q$ is an absolute constant. It is possible to deduce, from the above result, that if $f(z)$ is a real entire function which satisfies a linear differential equation whose coefficients are rational functions, and if $f(z)$ is bounded on the real axis, then every point of the real axis is a limit point of zeros of the successive derivatives of $f(z)$. (Received March 2,1955.)

\section{B. A. Fleishman: Periodic solutions of a nonlinear wave equa- tion. II.}

Let $L u \equiv u_{x x}-u_{t t}-2 \kappa u_{t}-\alpha u$, with $\kappa$ and $\alpha$ positive constants. Let $b(x, t)$ be such that $b(0, t)=0=b(\pi, t)$ and $b(x, t+p)=b(x, t)$. It was shown by the writer (Bull. Amer. Math. Soc. Abstract 59-4-365) that the initial value problem $L u=b+\epsilon u^{3}$ ( $\epsilon$ constant), $u(0, t)=0=u(\pi, t), u(x, 0)=f(x), u_{t}(x, 0)=g(x)$ has a solution $u(f, g ; x, t)$, and that $f$ and $g$ can be so chosen that $u$ is $p$-periodic in $t: u(f, g ; x, t+p)=u(f, g ; x, t)$, under the conditions that $\epsilon$ and $\left|\alpha-\kappa^{2}\right|$ be sufficiently small and, for given $p$, that $\alpha$ be sufficiently large (or, for given $\kappa$, that $p$ be sufficiently large). By using a more efficient integral operator, which inverts $L u$ rather than just $u_{x x}-u_{t t}$, and improving the proof in other respects, F. A. Ficken (Bull. Amer. Math. Soc. Abstract 60-2-225) obtained similar results without restriction on $\left|\alpha-\kappa^{2}\right|$. A detailed consideration of Ficken's result as applied to all multiples of the smallest period of $b(x, t)$, which themselves are periods, reveals that no requirements need be imposed on $\alpha, \kappa$, and $p$ except that they be positive. This work was supported by the Bureau of Ordnance, Department of the Navy, under Contract NOrd 7386. (Received January 13, 1955.)

\section{4t. K. O. Friedrichs: On differential forms.}

A theory of differential forms and harmonic fields on Riemannian manifolds, compact or with boundary, is developed, the operators $d$ and $\delta$ being treated directly by Hilbert space methods. The major tool is the non-invariant "complete" Dirichlet integral $D_{c}(v)$, essentially the integral of the sum of the squares of all first derivatives. Gaffney's inequality combined with the identity of the weak and strong extensions of $d$ and $\delta$ yields: If $v$ admits $d$ and $\delta$ weakly, $D_{c}(v)$ is finite and $D_{c}$ is finite for the exact, harmonic, and co-exact "components" of $v$. The complete continuity of $\int v * v$ with respect to $D_{c}(v)$ leads to the inequality $\int v * v \leqq c \int d v * d v+c \int \delta v * \delta v$ and then to the existence of the solution of $d \delta v=d f, \delta d v=\delta g$. Further it is proved: if $d v$ and $\delta v$ are bounded, the form $v$ is continuous and so are its three "components." In this approach the metric tensor is only required to have bounded first derivatives. For this reason the reduction by doubling of the theory for manifolds with boundaries to that for compact ones, described by Conner, can be carried out without specializing the metric tensor. Among the results for manifolds with smooth boundaries is the inequality $D_{c}(v) \leqq c \int d v * d v+c \int \delta v * \delta v$ for any form $v$ which is orthogonal to all harmonic 
fields. The methods differ from those employed by Morrey [Bull. Amer. Math. Soc. Abstracts 61-3-416] in a similar approach. (Received March 23, 1955.)

465. F. W. Gehring: Maximal theorems for solutions of the heat equation.

A well known theorem of Hardy and Littlewood (Acta Math. vol. 54) states that if $u(z)$ is harmonic in $|z|<1$, if $p>1$, and if $\int_{-\pi}^{\pi}\left|u\left(\rho e^{i \theta}\right)\right| P d \theta \leqq 1$ for $0 \leqq \rho<1$, then $\int_{-\pi}^{\pi} \mathrm{LUB}_{\rho}\left|u\left(\rho e^{i \theta}\right)\right|^{P} d \theta \leqq k(p)$. Similar results were obtained for functions harmonic in the half plane by Tims (J. London Math. Soc. vol. 27). Suppose that $u(x, t)$ is $C^{2}$ and that it satisfies the heat equation $u_{x x}=u_{t}$ in the half-plane $t>0$. Theorems similar to the following are proved. (I) If $p>1$ and if $\int_{-\infty}^{\infty}|u(x, t)|{ }^{p} d x \leqq 1$ for $t>0$, then $\int_{-\infty}^{\infty} \mathrm{LUB}_{t}|u(x, t)|^{p} d x \leqq k(p)$. (II) If $p>1$ and if $(1 / 2 X) \int_{-x}^{x}|u(x, t)|^{p} d x \leqq 1$ for $X$, $t>0$, then $(1 / 2 X) \int_{-X}^{x} \mathrm{LUB}_{t}|u(x, t)|^{p} d x \leqq k(p)$ for $X>0$. (Received March 2, 1955.)

466. J. L. Howell: Nonoscillation and integrability of solutions of a class of fourth-order differential equations.

Let $b(x)$ be positive and continuous on $(-\infty, \infty)$ and let $\lambda$ be positive. Then a necessary condition that $\left.\left(b y^{\prime \prime}\right)\right)^{\prime \prime}+\lambda y=0$ have a solution nonoscillatory as $x \rightarrow+\infty$ is that $x^{2}[b(x)]^{-1}$ be in $L(0, \infty)$. A sufficient condition that every solution be nonoscillatory for large $x$ is that $x^{\sigma} \int_{x}^{\infty} s^{2}[b(s)]^{-1} d s$ be bounded for some fixed $\sigma>1$. The necessary condition follows from consideration of the possible varieties of behavior of $\left(b y^{\prime \prime}\right)^{\prime}$ and is somewhat stronger than a similar condition deducible from a result of W. M. Whyburn (Amer. J. Math. vol. 52 (1930) pp. 176-178). The sufficient condition is obtained indirectly by iterating estimates of a supposed oscillatory solution. The equation has at most two solutions in $L(0, \infty)$ and at most two solutions in $L(-\infty, 0)$ but no solution in $L(-\infty, \infty)$. The same is proved with $L_{2}$ in place of $L$; then from a result of Glazman (Amer. Math. Soc. Translation No. 96, p. 17) it follows that there are exactly two solutions in $L_{2}(-\infty, 0)$ and exactly two in $L_{2}(0, \infty)$. (Received March 2, 1955.)

\section{H. K. Hughes and Ralph H. Niemann (p): A study of Stieltjes} integral transforms of a certain class of functions.

An extension of the well known factorial series $\sum_{n=0}^{\infty} a_{n} n ! \Gamma(z) / \Gamma(z+n+1)$ to the factorial transform $\int_{0}^{\infty} B(z, t+1) d \alpha(t)$ was considered by Fraser (Duke Math. J. vol. 11 (1944) pp. 469-486). Carmichael (Trans. Amer. Math. Soc. vol. 17 (1916) pp. 207232 ) found that the factorial series was a special case of a more general class of series, namely, $\sum_{n=0}^{\infty} c_{n} g(z+n) / g(z)$. An extension of this last series to the Stieltjes integral $\int^{\infty}[g(z+t) / g(z)] d c(t)$ is considered here. The function $g(z)$ is assumed to be analytic and single-valued in a sector of the complex plane and for values of $z$ of large modulus in this sector, $g(z)$ has the asymptotic representation $g(z) \sim z^{P(z)} e^{Q(z)}\left[1+\sum_{n=0}^{\infty} a_{n} / z^{n}\right]$ where $P(z)$ and $Q(z)$ are polynomials. The function $c(t)$ is assumed to be of bounded variation in every finite interval $(0, T)$. The region of convergence and of absolute convergence of the integral is in general a half-plane. Expressions for the boundary lines of these half-planes are found in terms of the functions $c(t)$ and $g(t)$. The integral converges uniformly in every bounded closed region in the interior of the half-plane of convergence and hence represents an analytic function there. Certain points may have to be excluded. An analytic continuation of the function defined by the integral is obtained when the function $c(t)$ is analytic. (Received March 7, 1955.) 
468t. Ernest Ikenberry: $A$ system of homogeneous spherical harmonics.

There is given a method for obtaining a basic set of h. s. h.'s: $Y_{i}=x_{i}, Y_{i j}=x_{i} x_{j}$ $-x^{2} \delta_{i j} / 3, \quad Y_{i j k}=x_{i} x_{j} x_{k}-3 x^{2} Y_{(i} \delta_{j k)} / 5, Y_{i j k l}=x_{i} x_{j} x_{k} x_{l}-6 x^{2} Y_{(i j} \delta_{k l)} / 7-x^{4} \delta_{(i j} \delta_{k l)} / 5$, etc., where $x^{2}=x_{1}^{8}+x_{2}^{2}+x_{3}^{2}$, and where $i, j$ and $k$ may each be assigned any of the values 1,2 or 3 . The parentheses around $s$ subscripts mean to sum over the $s !$ permutations of the subscripts and divide by $s$ !. The method gives a complete but not a minimal set of h. s. h.'s of any desired degree. Each h.s. h. of the set has the property that the contracted sum over any pair of indices is zero. The following contraction results are also noted: $x_{i} Y_{i}=x^{2}, x_{i} Y_{i j}=2 x^{2} Y_{j} / 3, x_{i} Y_{i j k}=3 x^{2} Y_{j k} / 5, x_{i} Y_{i j k l}=4 x^{2} Y_{j k l} / 7$, etc. (To be published in the American Mathematical Monthly.) (Received March 3, 1955.)

469t. W. B. Jurkat: An extension problem for functions with monotonic derivatives.

Let $F(x)$ be the $n$th integral of a positive nondecreasing function for all large positive $x$; the problem to be solved is to find a function $f(x)$, being the $n$th integral of a nondecreasing function for all $x$, with the property $f(x)=F(x)$ for all large positive $x$, $f(x)=0$ for all large negative $x$. A complete solution of this and connected boundary value problems is given by means of the theory of moment problems. The main result of the paper consists of determining the behaviour of certain quadratic forms, on which the solution depends. (Received January 12, 1955.)

\section{Tosio Kato: Note on Friedrichs extensions.}

The following theorem will be proved. Let $J_{1}, J_{2}$ be two closed quadratic forms bounded from below with dense domains in a Hilbert space $R$. Let $J_{1} \subset J_{2}$ and let $m \equiv \operatorname{dim}$ (Domain $J_{2} /$ Domain $J_{1}$ ) $<\infty$. Let $H_{1}, H_{2}$ be the self-adjoint operators associated with $J_{1}, J_{2}$ respectively. Then the intersection $D$ of the domains of $H_{1}$ and $H_{2}$ is dense in $R$ and $H_{1}$ and $H_{2}$ coincide in $D$. The common restriction $H_{0}$ of $H_{1}$ and of $H_{2}$ to domain $D$ is a symmetric operator bounded from below with deficiency indices $(m, m)$, and the Friedrichs extension of $H_{0}$ coincides with $H_{1}$. This theorem is often convenient in determining the Friedrichs extension of a given symmetric operator bounded from below. For instance let $R=L^{2}(0,1)$ and $H_{0}=-d^{2} / d x^{2}$ with the boundary condition $u(0)=u^{\prime}(0)=u(1)=u^{\prime}(1)=0$. Then the above theorem is applicable if we take as $H_{1}, H_{2}$ the differential operators $-d^{2} / d x^{2}$ with the boundary conditions $u(0)=u(1)=0$ and $u^{\prime}(0)=u^{\prime}(1)=0$ respectively, showing that $H_{1}$ is the Friedrichs extension of $H_{0}$. (Received March 1, 1955.)

\section{G. L. Krabbe: Certain abelian rings of operators on $l_{p}$.}

Suppose $p>1$ and let $l_{p}$ denote the Banach space of all sequences $c$ such that $\sum_{n=-\infty}^{\infty}\left|c_{n}\right|^{p}<\infty$. To any function $A$ corresponds a transformation $A_{*}$ defined for any $c$ in $l_{p}$ by $A_{*} c=b, b_{n}=\sum_{\nu-\infty}^{\infty} a_{n-\nu} c_{\nu}, n=0, \pm 1, \pm 2, \cdots$, with the understanding that $a$ is the sequence of Fourier coefficients of $A$. If $A$ is in the set $\mathscr{B}$ of all functions of bounded variation on $[-\pi, \pi]$, then $A_{*}$ is a bounded operator on $l_{p}$ [Steckin, Doklady Akad. Nauk. SSSR, N. S. vol. 71 (1950) pp. 237-240]. Theorem: The sets $\mathfrak{B}$ and $\mathfrak{B}_{*}=\left\{A_{*} \mid A \in \mathfrak{B}\right\}$ are isomorphic abelian rings under composition; thus $(A B)_{*}$ $=A_{*} B_{*}$ when $A \in \mathfrak{B}$ and $B \in \mathfrak{B}$. Let $\mathfrak{A}$ be the set of all functions analytic in some circle $\{\lambda|| \lambda \mid<r\}, r>\pi$; then $\mathfrak{A}_{*}=\left\{A_{*} \mid A \in \mathfrak{A}\right\}$ forms an irreducible subring of $\mathfrak{B}_{*}$. The spectrum of any $A_{*}$ in $\mathfrak{A}_{*}$ is the image under $A$ of the interval $[-\pi, \pi] ; A_{*}$ has 
no eigenvalues when $A$ is one-to-one. This generalizes corresponding results of Toeplitz [F. Riesz, Les systemes d'équations linéaires a une infinité d'inconnues, Paris, 1913, pp. 175 and 153]. (Received December 20, 1954.)

\section{2t. G. L. Krabbe: On the spectra of certain operators on $l_{p}$.}

Let $I$ denote the identity-function $I(\lambda)=\lambda$. Then $I_{*}$ is the Hilbert-transformation on $l_{p}$ (see the preceding abstract for definitions). The ring $\mathfrak{A}_{*}$ coincides with the ring $\left\{A\left(I_{*}\right) \mid A \in \mathfrak{A}\right\}$, in fact $A_{*}=A\left(I_{*}\right)$ whenever $A \in \mathfrak{A}$ (the symbol $A\left(I_{*}\right)$ is to be interpreted in the sense of the Dunford operational calculus). Let $E(\lambda)=\exp (-i \lambda)$; the spectrum $\sigma\left(E_{*}\right)$ of $E_{*}$ is exactly the circumference of the unit-circle, and $\sigma\left(I_{*}\right)$ $=[-\pi, \pi]$. The point-spectrum of both operators is empty. Therefore $I_{*}=i \log E_{*}$ in the sense specified in a previous article (On the logarithm of uniformly bounded operators, Bull. Amer. Math. Soc. Abstract 60-6-708), although $\log E_{*}$ is not definable by the Dunford calculus. (Received December 20,1954.)

473t. Erwin Kreyszig: On solutions of certain partial differential equations of elliptic type.

The Bergman integral operator (1) $\int_{-1}^{1} E\left(z, z^{*}, t\right) f\left(z\left(1-t^{2}\right) / 2\right)\left(1-t^{2}\right)^{-1 / 2} d t$, where the generating function $E$ of (1) satisfies (2) $\left(1-t^{2}\right) E_{z^{*} t}-\left(E_{z^{*}} / t\right)+2 z t L(E)=0$, $L(E)=E_{z z^{*}}+B E_{z^{*}}+C E, \quad E_{z^{*}} \equiv \partial E / \partial z^{*}, z=z_{1}+i z_{2}, z^{*}=z_{1}-i z_{2}$, transforms analytic functions $f(z)$ of one complex variable (regular at the origin) into solutions $u\left(z, z^{*}\right)$ of partial differential equations (3) $L(u)=0$ of elliptic type (Bergman, Rec. Math. N.S. vol. 2 (1937) p. 1169, Duke Math. J. vol. 6 (1940) p. 537). (The general equation $u_{z z^{*}}+a u_{z}+b u_{z^{*}}+c u=0$ can be always reduced to the form (3).) For every equation (3) various functions $E$ exist. For different purposes different $E$ are advantageous. Of special interest are functions $E$ generating solutions of (3) which satisfy an ordinary differential equation in addition to (3), since then the theory of these equations yields us new tools for investigating solutions of (3). The generating functions "of exponential type" (4) $E=\exp \left(\sum_{\mu=0}^{m} g_{\mu}\left(z, z^{*}\right) t^{\mu}\right)$ corresponding to a large class of equations (3) have this property: If $f(z)=z^{n}, n=0,1, \cdots$, the functions (1) generated by (4) satisfy the ordinary linear differential equation (5) $\sum_{\kappa=0}^{k} b_{k-k} u^{(k-\kappa)}=0, b_{k}=1$, with respect to $z_{1} . k$ is independent of $n$ and $k \leqq m+1$. The $b_{\mu}$ have poles of order $m+1-\mu$ at the point $z=0 ;(5)$ is therefore of Fuchs type at $z=0$. (Received March 17, 1955.)

\section{4t. Erwin Kreyszig: On singularities of certain linear partial dif- ferential equations.}

Solutions $u\left(z, z^{*}\right)$ of (1) $u_{z z^{*}}+B u_{z^{*}}+C u=0$ generated by Bergman's integral operator in the form $(2) u\left(z, z^{*}\right)=\int_{-1}^{1} E\left[(z / 2)^{n}\left(1-t^{2}\right)^{n-1 / 2}\right] d t$ where the generating function (3) $E=\exp \left(\sum_{\mu=0}^{m} q_{\mu}\left(z, z^{*}\right) t^{\mu}\right)$ is of exponential type satisfy also an ordinary differential equation (see the previous note). These functions (2) correspond to a large class of equations (1) characterized in the following theorems: I. If the coefficients of (1) are of the form $B=-q_{0, z}-q_{2} / 2 z, q_{0, z} \equiv \partial q_{0} / \partial z, C=-q_{1} q_{1, z^{*}} / 2 z$ where $q_{0}=q_{0}(z), \quad q_{1}=\sum_{\nu=0}^{\sigma(m)} a_{\nu} z^{\nu+1 / 2}, \quad a_{0}=a_{0}\left(z^{*}\right), \quad a_{\nu}=$ const., $\quad \nu=1,2, \cdots, \quad \sigma(m), \quad \sigma(m)$ $=(m / 2)-1(m$ even $)$ or $\sigma(m)=(m-1) / 2(m$ odd $), q_{2}=\sum_{\nu=1}^{\tau(m)} d_{\nu} z^{\nu}, d_{\nu}=$ const.; $\tau(m)$ $=m / 2(m$ even $)$ or $\tau(m)=(m-1) / 2(m$ odd $)$, then the solutions of $(1)$ can be generated by (3) and $q_{2 \mu+1}=\left((-2)^{\mu} / 3 \cdot 5 \cdots(2+1)\right) \sum_{\nu=\mu}^{\sigma(m)} \nu(\nu-1)(\nu-2) \cdots(\nu-\mu+1) a_{\nu} z^{\nu+1 / 2}$, $\mu=1,2, \cdots, \sigma(m), q_{2 \mu}=-\left((-2)^{\mu} / 2 \cdot 4 \cdots 2 \mu\right) \sum_{\nu=\mu}^{\tau(m)}(\nu-1)(\nu-2) \cdots(\nu-\mu+1) d_{\nu} z^{\nu}$, $\mu=2,3, \cdots, \tau(m)$. II. If $B$ can be written as in I and $C=-q_{2, z^{*}} / 2 z$, then the same holds, but in this case one has $q_{1}=0$ (consequently $q_{3}=q_{5}=\cdots=0$ ) and $d_{1}$ can be 
a function of $z^{*}$. III. If the generating function $E$ is of exponential type no possibilities for $B$ and $C$ exist other than those indicated in I and II. In consequence of these theorems which include results of Bergman (Rec. Math. N.S. vol. 2 (1937) p. 1169), Nielsen and Ramsey (Bull. Amer. Math. Soc. vol. 49 (1943) p. 156) there exist simple relations between singularities of the coefficients of (1) and those of $q_{0}, q_{1}, q_{2}$. (Received March 17, 1955.)

475t. P. D. Lax: Differentiability of solutions of partial differential equations. I. Preliminary report.

The Cauchy problem for symmetric hyperbolic systems $A u=v$ in the sense of Friedrichs (Comm. Pure Applied Math. (7) (1954) pp. 345-392) can be reduced to the problem of finding a periodic solution of $A u=v$. A weak periodic solution can be constructed by projection, about which the following differentiability theorem is proved: If $\|v\|<\infty,\left\|u_{s}\right\|<\infty, s>0$. Here $\|v\|_{s}$ denotes the $s$-fold Dirichlet integral for functions with $s$ strong derivatives. We also introduce the negative norm $|v|_{-s}$ $=\operatorname{Sup}_{w}(v, w) /\|w\|_{8 .}$ The tools for proving the differentiability are: (i) the a priori estimate $\|a\|_{-s} \leqq\|b\|_{-s}$ for the adjoint equation $A^{*} a=b$; (ii) the denseness of the range $A^{*} a=b, a$ smooth, in the norm $\|b\|_{-\rightarrow}$; (iii) the lemma that if $(u, b) \leqq\|b\|_{-s}$, then $\|u\|_{s}<\infty$. (i) is proved by constructing a $c$ such that $\Delta^{8} c=a$ and integrating by parts the left side of $\left(c, A^{*} \Delta^{s} c\right)=(c, b)$, obtaining $\|c\|_{s} \leqq\|b\|_{-s \cdot}$. (ii) follows from the boundedness of $A$ from below, and (iii) is just a variant of Friedrichs' theorem about the identity of weak and strong differentiability. (Received March 21, 1955.)

\section{P. D. Lax: Differentiability of solutions. II.}

Distribution solutions of $L u=v, L$ an elliptic operation of order $2 m$, have this differentiability property: if $\|v\|_{s-m}$ is finite over a subdomain $D$, so is $\|u\|_{s+m}$ over any subdomain. This generalizes a result of Friedrichs (Comm. Pure Applied Math. (6) (1953) pp. 299-326; see there the differentiability theorems of L. Schwartz, F. Browder, L. Gårding and F. John). The theorem is proved first in the periodic case, by these tools: (i) the a priori estimate $\|a\|_{m+s} \leqq\|b\|_{s-m}$ for the adjoint equation $L^{*} a=b, s \leqslant 0$; (ii) the denseness of the range of $L^{*} a=b, a$ smooth, in the norm $\|v\|_{s-m}$; (iii) The identity of weak and strong differentiability. (i) follows for $s \geqq 0$ from Gårding's inequality, for $s \leqq 0$ from Gårding's inequality applied to the operator $L^{*} \Delta^{s}$ and the function $c$ defined by $\Delta^{s} c=a$; (ii) follows for $s \leqq 0$ from Garding's inequality, for $s>0$ from Garding's inequality applied to $\Delta^{*} L^{*}$. The passage from the periodic to the nonperiodic case is accomplished by observing that $u^{\prime}=\phi u, \phi$ compactly carried over $D$, can be regarded as periodic solution of $L u^{\prime}=\phi u+N u, N$ of degree $2 m-1$, and by doing a finite induction. (Received March 21, 1955.)

\section{Mark Lotkin: On the accuracy of the adjoint method of differen- tial corrections.}

When solving systems of ordinary differential equations it is of ten desirable to obtain also estimates of the effect of small perturbations upon the solutions. For the prediction of such small effects there is frequently employed the "method of adjoints," especially in ballistics and related fields. Now the system of adjoint differential equations involves the calculation of certain partial derivatives, which, especially if empirical functions occur in the original system of differential equations, are not always computable with sufficient accuracy. The problem discussed is thus con- 
cerned with the degree of accuracy required of the quantities occurring in the original system in order to guarantee that the differential effects will stay below a prescribed bound. The conclusions are found to lead to quite useful answers. (Received January $13,1955$.

\section{John McCarthy: Synchronization and the rotation number.}

Let the continuous function $f(x, \lambda)$ satisfy $f(x+1, \lambda)=f(x, \lambda)+1$ and for fixed $x$ increase monotonically from $-\infty$ to $+\infty$ with $\lambda$. Letting $x_{n+1}=f\left(x_{n}, \lambda\right)$ and $x_{0}$ arbitrary the Poincaré rotation number is $\nu(\lambda)=\lim x_{n} / n$ and is independent of $x_{0}$. The author considers the function $\nu(\lambda)$ and shows that it is continuous and monotonic as a function of $\lambda$. It is further shown that in general (for $f(x, \lambda)$ in a second category set in the topology of uniform convergence) for each rational number $p / q$ there are numbers $\lambda_{1}$ and $\lambda_{2}$ such that for $\lambda_{1} \leqq \lambda \leqq \lambda_{2}, \nu(\lambda)=p / q$. Thus in general $\nu(\lambda)$ does all its increasing on a set of the first category. The preceding results are applied to get a theory of rotated vector fields on the torus and to indicate that in general a physical system depending on a parameter will have periodic solutions for a set of parameter values of the second category. (Received March 7, 1955.)

\section{9t. E. J. McShane: On Stieltjes integration.}

For the Stieltjes integral $\int f(x) d g(x)$ in one dimension the following theorems are well known. (I) If the integral exists, the integrand $f$ and the interval function $\Delta_{0}$ can have no common discontinuity. (II) If the integral exists and $g$ is of bounded variation, then $f$ is also integrable with respect to the total variation of $g$. In this note these two theorems are extended to $n$-space, both for the ordinary Stieltjes integral and for the modified form of it defined by McShane and Botts. (Received March 1, 1955.)

480. Paul Malliavin: On the maximum modulus of a function of exponential type in a half-plane.

Let $f(z)$ be a holomorphic function in $x>0$, satisf ying $\lim \sup (\log |f(i y)|) /|y| \leqq d \pi$, $L$ a sequence of positive numbers verifying $\left|l-l^{\prime}\right|>h>0, l \neq l^{\prime}$; denote by $l(r)$ $=\sum_{l<r} 1 / l(l \in L)$, by $D_{*}(L)=\lim \inf _{a-\infty, b / a=\infty}(l(b)-l(a)) /(\log b-\log a)$, by $D^{*}(L)$ the superior limit of the same expression. Then a necessary and sufficient condition in order that $\lim _{L}(\log |f(l)|) / l=-\infty$ imply $\lim (\log |f(x)|) / x=-\infty$ is that $d$ $<D_{*}(L)$; furthermore if this condition is fulfilled there exists a constant $A$ depending only on $L$ and $d$ such that $A+\lim \sup _{L}(\log |f(l)|) / l>\lim \sup (\log |f(x)|) / x$. As an application one gets the following gap result on the analytic prolongation of Taylor series: $M$ denoting a sequence of integers there exists a function $h(u)=\sum a_{m} u^{m}$ $(m \in M)$ which can be prolonged outside its circle of convergence to infinity inside the angle $|\arg u|<\pi c$ if and only if $D^{*}(M)>c$. As corollary if one takes two sequences $M_{1}$ and $M_{2}$ whose reunion is the set of all integers and satisfying $D^{*}\left(M_{1}\right)$ $=D^{*}\left(M_{2}\right)=1$, one gets two functions $h_{1}(u)$ and $h_{2}(u)$, each of them being holomorphic in the plane cut by the half line $(-1,-\infty)$, their Taylor series in 0 having complementary exponents. (Received March 3, 1955.)

\section{Joseph Milkman: Logarithmic sequences.}

A logarithmic sequence is a set of $n$ real numbers $a_{i}, i=1,2, \cdots, n$, such that (1) $0 \leqq a_{i} \leqq 1$; (2) $a_{k}+a_{l}<a_{r}+a_{s}$ if $k l<r s$ and (3) $a_{k}+a_{l}=a_{r}+a_{s}$ if $k l=r s$. For $r \leqq n$ and $s \leqq n$ form all the different products $r s$. The sequence $p_{1}, p_{2}, \cdots, p_{t}$ is the sequence 
of all these distinct products arranged in increasing order. Corresponding to $p_{i}=r s$ define $s_{i}=a_{r}+a_{s}$ and $\Delta_{i}=s_{i+1}-s_{i}>0$. Min $\Delta(a)$ denotes the smallest $\Delta_{i}$ for a given logarithmic sequence $a_{i} . m_{1}, m_{2}, \cdots, m_{n}$ is a maximizing sequence if it is a logarithmic sequence and $\min \Delta(m) \geqq \min \Delta(a)$ for all logarithmic sequences $a_{1}, a_{2}, \cdots, a_{n}$. If one represents the logarithmic sequences by points $\left(a_{1}, \cdots, a_{n}\right)$ in $n$-dimensional Euclidean space, the set $L$ of all points representing logarithmic sequences is convex. A maximizing sequence of order $n$ exists for every natural number $n$ and can be obtained by a finite number of operations. The author has computed the maximizing sequences for $n=1,2, \cdots, 7$ and found them to be unique and rational. (Received March 3, 1955.)

482. C. B. Morrey, Jr.: A variational method in the theory of harmonic integrals. II.

The variational method introduced in part I is applied to the study of differential forms and boundary value problems on a compact Riemannian manifold with boundary $B$. The manifold is not assumed to be orientable and parallel theories are developed for even and odd forms. Complete results, analogous to those for a compact manifold, are first obtained for the subspaces $\mathfrak{P}_{2}^{+}$of $\mathfrak{P}_{2}$ where the normal part $n \omega=0$ on $B$, and $\mathfrak{B}_{2}^{-}$where the tangential part $t \omega=0$ on $B$. By using the results about special potentials in these spaces, an orthogonal decomposition theorem $\mathfrak{R}_{2}=\mathfrak{H} \oplus \mathfrak{B} \oplus \mathfrak{D}$ is probed; $\mathfrak{A}$ consists of all harmonic fields, $\mathfrak{B}$ consists of all elements $\delta \alpha$ for $\alpha \in \mathfrak{P}_{2}^{+}$, and $\mathcal{D}$ consists of all elements $d \beta$ where $\beta \in \mathfrak{P}_{2}^{-}$. We may take $\alpha=d \Omega, \beta=\delta \Omega$ where $\Omega$ is a "free" potential of $\omega-H$ ( $\omega=$ given form) in which $n d \Omega=t \Omega=0$. Using this decomposition, the boundary value results for harmonic fields and forms recently obtained by Duff and Spencer (Ann. of Math. (1952)) and Conner (Proc. Nat. Acad. Sci. U.S.A. (1954) pp. 1151-1155) are obtained immediately. Complete differentiability results are obtained on manifolds of class $C_{\mu}^{k} \geqq C_{1}^{1}$. (Received March 3,1955.)

\section{A. E. Nussbaum: The Bernstein-Hausdorff-Widder theorem for locally compact abelian semi-groups.}

Let $\left\{T_{x}\right\}_{x \in \gamma}$ be a semi-group of uniformly bounded self-adjoint operators in a Hilbert space. $\gamma$ is a semi-group contained in a locally compact abelian group $\mathfrak{g}$ and integrable with respect to the Haar measure of $\mathfrak{g}$. A real character of $\gamma$ is defined to be a continuous homomorphism of $\gamma$ into the multiplicative semi-group of real numbers. The real characters $\chi(x)$ of $\gamma$ form a semi-group $\gamma^{x}$, the real character semi-group of $\gamma$, topologized by the weakest topology in which the mappings $x: \chi \rightarrow \chi(x)$ are continuous. It is shown that there exists a spectral measure $E(\sigma)$ with respect to the Borel subsets $\sigma$ of $\gamma^{x}$ such that $T_{x}=\int_{\gamma} x(x) d E(\sigma)$. The method of proof is that used by R. S. Phillips (Trans. Amer. Math. Soc. vol. 71 (1951)) in his demonstration of Stone's theorem on groups of unitary operators for locally compact abelian parameter groups. Using this theorem and reproducing kernel spaces it is shown how it yields the Bernstein-Hausdorff-Widder theorem on the representation of completely monotonic functions on locally compact abelian semi-groups by generalized Laplace-Stieltjes integrals: A function $f(x)$ on $\gamma$ is completely monotonic if and only if there exists a non-negative measure $d \alpha(\sigma)$ with respect to the Borel subsets $\sigma$ of $\gamma^{x}$ whose spectrum is contained in the set $\gamma_{0}=\{\chi \mid 0 \leqq \chi(x) \leqq 1\}$ such that $f(x)$ $=\int_{\gamma \circ \chi}(x) d \alpha(\sigma)$. (Received March 3, 1955.)

484. Robert Osserman: $A$ hyperbolic surface in 3-space. 
Using the observation that a simply-connected Riemann surface which contains two intersecting lines of symmetry and a third disjoint from these must be hyperbolic, a hyperbolic surface of the form $z=f(x, y)$ is constructed, where $f(x, y)$ is defined, single-valued, and continuous over the whole $(x, y)$-plane. (Received March 3, 1955.)

\section{5t. L. B. Robinson: On a complete system of semitensors obtained by noncommutative symbolic multiplication.}

The author has been able to compute some complete systems of tensors and semitensors with the aid of commutative symbolic multiplication. Now he has computed a complete system of semitensors with the aid of noncommutative symbolic multiplication. He will give the formula for this system. Write $f_{11} \equiv y_{1}^{2} I_{22}-y_{1} y_{2} I_{12}-y_{1} y_{2} I_{21}+y_{2}^{2} I_{11}$ $=\Psi_{11}, \quad f_{12} \equiv y_{1} Y_{1} I_{22}-y_{2} Y_{1} I_{12}-y_{1} Y_{2} I_{21}+y_{2} Y_{2} I_{11}=\Psi_{12}, \quad f_{21} \equiv y_{1} Y_{1} I_{22}-y_{1} Y_{2} I_{12}-y_{2} Y_{2} I_{21}$ $+y_{2} Y_{2} I_{11}=\Psi_{21}, f_{22} \equiv Y_{1}^{2} I_{22}-Y_{1} Y_{2} I_{12}-Y_{1} Y_{2} I_{21}+Y_{2}^{2} I_{11}=\Psi_{22}$. The $\Psi$ are arbitrary functions of the covariants calculated by Wilczynski. If one solves this system with respect to $I_{i j}$ one has the complete system of semitensors. (Received March 3, 1955.)

486t. Walter Rudin: Maximal subalgebras in spaces of continuous functions.

For any compact Hausdorff space $X$, let $C(X)$ denote the Banach algebra of all complex continuous functions on $X$. A subalgebra of $C(X)$ is called maximal if it not a proper subset of any closed proper subalgebra of $C(X)$. The existence of maximal subalgebras of $C(L)$ (other than maximal ideals), where $L$ is a simple closed curve, has been demonstrated by Wermer (Proc. Amer. Math. Soc. vol. 4 (1953) pp. 866869; Bull. Amer. Math. Soc. Abstract 61-1-51). The following theorem is now proved. If $X$ is a compact Hausdorff space which contains a subset $K$ homeomorphic to the Cantor set, then $C(X)$ contains a maximal closed subalgebra $M_{K}$ which separates points on $X$; every $f \in M_{K}$ has the property that the boundary of the unbounded component of the complement of $f(K)$ is connected. (Received February 24, 1955.)

487. Walter Rudin: Uniform approximation by polynomials in two complex variables.

Let $E$ be an uncountable compact set of real numbers, and let $R(f, g)$ be the uniformly closed algebra (containing the complex constants) generated by the complex continuous functions $f$ and $g$ on $E$; it is assumed that $R(f, g)$ separates points on $E$. Of the following three statements, the first is well known, the other two are proved here. (1) If $f$ and $g$ are real, then $R(f, g)$ contains every continuous complex function on $E$. (2) The same is true if only one of the two generating functions is required to be real. (3) The conclusion is false if neither $f$ nor $g$ are required to be real. If $E$ is an interval, (3) solves a problem posed by Mergelyan (Amer. Math. Soc. Translation No. 101, p. 29). A statement equivalent to (3) is that there exists a set $F$, homeomorphic to $E$, in the space of two complex variables $z$, $w$, and a complex continuous function $h$ on $F$ which cannot be approximated uniformly on $F$ by polynomials in $(z, w)$. (Received February 22, 1955.)

\section{8t. I. E. Segal: Tensor algebras over Hilbert spaces. II.}

The system of all skew-symmetric tensors over a complex Hilbert space $H_{c}$ is treated here from the standpoint of an earlier corresponding treatment of the system of symmetric tensors. Either system is canonically unitarily equivalent to the space 
$L_{2}(H)$ relative to a generalized type of probability distribution on the real Hilbert space $H$ of which $H_{c}$ is the complex extension. In the skew-symmetric case the appropriate distribution is however of a "noncommutative" type, and in fact $L_{2}(H)$ is the Clifford algebra over $H$ when $H$ is finite-dimensional and is otherwise the $L_{2}$-system of a factor of type $\mathrm{II}_{1}$. In either case the canonical equivalence takes the canonical field $Q$-operators into simple multiplication operators. It is shown that from a pure probability viewpoint also the Clifford distribution relevant to the skew-symmetric tensors is closely related to the normal distribution relevant to the symmetric tensors. (Received February 24, 1955.)

\section{9t. V. L. Shapiro: Generalized Laplacians and the Abel summation} of multiple trigonometric series.

Letting $x=\left(x_{1}, \cdots, x_{n}\right), y=\left(y_{1}, \cdots, y_{n}\right), \alpha x+\beta y=\left(\alpha x_{1}+\beta y_{1}, \cdots, \alpha x_{n}+\beta y_{n}\right)$, $(x, y)=x_{1} y_{1}+\cdots+x_{n} y_{n}$, and $|x|=(x, x)^{1 / 2}$ and denoting the surface of the $n$-dimensional sphere with center $x_{0}$ and radius $t$ by $C_{n}\left(x_{0}, t\right)$, the author sets $L\left(F, x_{0}, t\right)$ $=\omega_{n}^{-1} \int_{C_{n}(0,1)} F\left(x_{0}+t x\right) d S_{n-1}(x)$ where $\omega_{n}$ is the $(n-1)$-dimensional volume of $C_{n}(0,1)$ and $d S_{n-1}(x)$ is its $(n-1)$-dimensional volume element. He next sets $\nabla\left(F, x_{0}, t\right)$ $=L\left(F, x_{0}, t\right)-F\left(x_{0}\right)$ and $\psi^{*} F\left(x_{0}\right)=\lim \sup _{t \rightarrow 0} 2 n \nabla\left(F, x_{0}, t\right) t^{-2}$ with a similar definition involving lim inf for $\psi_{*} F\left(x_{0}\right)$ and calls $\psi^{*} F\left(x_{0}\right)$ and $\psi_{*} F\left(x_{0}\right)$ the upper and lower generalized Laplacians of $F$ at the point $x_{0}$, respectively. The multiple trigonometric series $S=\sum a_{m} e^{i(m, x)}$ is said to be Abel-summable to $f(x)$ if $\lim _{z \rightarrow 0} \sum a_{m} e^{i(m, x)} e^{-|m|_{z}}$ $=f(x) . S$ will be called a real-valued multiple trigonometric series if $a_{m}=\bar{a}_{-m}$. The following theorem is then proved: Let $f^{*}(x)$ and $f_{*}(x)$ be the upper and lower Abel sums of the real-valued multiple trigonometric series $S=\sum a_{m} e^{i(m, x)}$. Suppose that $C-\sum_{m \neq 0} a_{m}|m|^{-2} e^{i(m, x)}$ is the Fourier series of a continuous periodic function $F(x)$. Then for all $x$ the following inequalities hold: (a) $\psi_{*} F(x) \leqq f^{*}(x)$ and (b) $f_{*}(x) \leqq \psi^{*} F(x)$. This result enables one to extend many of the previous results on the uniqueness of multiple trigonometric series involving spherical $(C, 1)$ summability to those involving Abel summability. (Received February 23, 1955.)

\section{Wilhelm Stoll: Reconstruction of meromorphic modifications by} $\sigma$-processes.

If $G$ and $H$ are complex manifolds of dimension 4, if $\tau$ pseudoconformally maps the open subset $A \subseteq G$ onto $B \subseteq H$, if $M=G-A$ and $N=H-B$ lie in analytic sets of dimension $2 p \leqq 2$, then $\mathfrak{M}=\mathfrak{M}(G, H, M, N, \tau)$ is a modification. $\mathfrak{M}$ is open in both directions if $\tau(U \cap A) \cup_{N}$ and $\tau^{-1}(V \cap B) \cup M$ are open for all open sets $U \supseteq M$ and $V \supseteq N . \mathfrak{M}$ is meromorphic if for each 2-dimensional complex submanifold $L \subset G$, with $L \cap M=\left\{P_{0}\right\}$, the set of all accumulation points of all sequences $\tau\left(P^{\nu}\right)$ with $P^{\nu} \in L \cap A$ and $\lim _{\nu \rightarrow \infty} P_{\nu}=P_{0}$ consists of at most one point. To each 4-dimensional complex manifold $G$ and each point $P \in G$ there is one and-up to analytic equivalence -only one modification $S_{P}=\mathfrak{M}\left(G, H_{P},\{P\}, N_{P}, \sigma_{P}\right)$ called a $\sigma$-process, where $N_{P}$ is an analytically imbedded 2 -sphere. When $\mathfrak{M}$ is a modification, which is open in both directions and meromorphic, each singularity $P \in G$ of $\tau$ is replaced by such a sphere, and then each singularity of $\tau \sigma_{P}^{-1}$ by such a sphere, and so on. One gets a tree of $\sigma$-processes approaching a limit manifold $F$. Analogously, with respect to $H$ and $\tau^{-1}$, one gets a second tree approaching the same manifold $F$. The natural projections $\omega: F \rightarrow G$ and $\chi: F \rightarrow H$ are maps onto. On $A, \tau=\chi \omega^{-1}$. Hence, the given modification is reconstructed by $\sigma$-processes and inverse $\sigma$-processes. (Received March 1 , 1955.) 


\section{Seth Warner: Inductive limits of normed algebras.}

A subset $B$ of a locally multiplicatively-convex algebra $E$ is called idempotently bound if for some nonzero scalar $\lambda, \lambda B$ is contained in a bound, idempotent set; $E$ is called pointwise idempotently bound if every point is an idempotently bound subset. A transformation defined on $E$ is idempotently bound if it maps idempotently bound subsets into bound subsets. Defining the algebraic inductive limit topology on $E$ with respect to locally $m$-convex algebras $E_{\alpha}$ and homomorphisms $g_{\alpha}: E_{\alpha} \rightarrow E$ in an obvious way, one obtains the following three equivalent conditions on $E$ : (1) $E$ is the algebraic inductive limit of normed algebras; (2) Every convex, equilibrated, absorbing set absorbing all idempotently bound subsets is a neighborhood of zero; (3) Every idempotently bound homomorphism from $E$ is continuous. Algebras satisfying these conditions are called ibic algebras. If $E$ is commutative and metrizable, then $E$ is a pointwise idempotently bound, ibic algebra if and only if $\left[x \mid x^{n} \rightarrow 0\right]$ is a neighborhood of zero. If $E$ is a complete, commutative locally $m$-convex algebra over the complex numbers, every multiplicative linear functional is idempotently bound. Many algebras drawn from integration theory and the theory of distributions, including the space $(D)$ of Schwartz, are ibic algebras. (Received March 1, 1955.)

492t. František Wolf: On selfadjoint differential equations with nonselfadjoint boundary conditions. Preliminary report.

If $A$ is closed and $\left\|\left(A-\lambda_{0} I\right) f\right\| \geqq M\|f\|$ for all $f \in D_{A}$, def $\left(A-\lambda_{0} I\right)=m<\infty$ and $A_{1}, A_{2}$ two extensions such that $\lambda_{0}$ is in the resolvent set of both, then $\left(A_{1}-\lambda_{0} I\right)^{-1}$ $-\left(A_{2}-\lambda_{0} I\right)^{-1}$ is an operator with $m$-dimensional range. To this finite-dimensional perturbation can be applied the results of D. C. Kleinecke (O.O.R. report 1953, University of California at Berkeley). These perturbed eigenvalues are roots of a meromorphic function given as a determinant whose elements can be computed, if we know the resolvent of the unperturbed operator. In this case the unperturbed problem is a selfadjoint boundary problem, the perturbed problem is of the type mentioned in the title. Knowing the eigenvalues, the eigenfunctions can be obtained by applying the unperturbed resolvent to known functions. (Received March 1, 1955.)

\section{Applied Mathematics \\ 493t. Milton Abramowitz: Forced heat convection in laminar flow through a tube.}

The problem of steady state heat transfer in a tube is discussed. The assumption that the mean velocity is so large that axial temperature variations are negligible is discarded and the equation of heat transfer is then solved by the method of Laplace transform. The resulting eigenvalue problem is then discussed and expansions are obtained for the eigenfunctions and eigenvalues. (Received January 24, 1955.)

\section{4t. R. G. Blake: Polynomial solutions in laminated orthotropic plane strain.}

This paper considers exact polynomial solutions of the problem of plane strain in a rectangle consisting of three layers of orthotropic material cemented together, with the two outer layers similar. The lines of separation are taken parallel to the $x$-axis and in the $x y$-plane. Using $\tau_{i j 1}$ for the stresses in the central layer and $\tau_{i j 2}$ for the stresses in the outer layer, it is shown that the only exact polynomial solutions are (1) $\tau_{x x 1}=k_{1}, \tau_{x x 2}=k_{2}, \tau_{y y 1}=\tau_{y y 2}=k_{3}, \tau_{x y 1}=\tau_{x y 2}=0$ (ki constant); (2) $\tau_{x x 1}=\tau_{x x 2}$ 
$=\tau_{y y 1}=\tau_{y y 2}=0, \tau_{x y 1}=\tau_{x y 2}=k$ ( $k$ constant); (3) $\tau_{x x 1}=k_{1} x y, \tau_{x x 2}=k_{2} x y, \tau_{y y 1}=\tau_{y y 2}=0$, $\tau_{x y 1}=k_{3} y^{2}+k_{4}, \tau_{x y 2}=k_{5} y^{2}+k_{6}$ ( $k_{i}$ constant). This research was supported by a contract with the Office of Naval Research. (Received February 4, 1955.)

\section{J. R. Isbell: Pseudo-main solutions.}

H. Gurk defines (unpublished) a main solution of an $n$-person game as a finite solution determined by a family $U$ of minimal winning sets and a system $\left(x_{i}\right)$ of weights for the players; the solution $V_{U}$ is $\left\{\alpha^{S} \mid S \in U\right\}$, where $\alpha_{i}^{s}$ is $x_{i}$ for $i$ in $S, 0$ for $i$ not in $S$. He defines a pseudo-main solution as a solution $\left\{\alpha^{s}\right\} \cup W$, where $\left\{\alpha^{S}\right\}$ would be a main solution except that the finite set $W$ is undominated. The author defines (A class of game solutions, Proc. Amer. Math. Soc. vol. 6 (1955)) pseudo-main solutions as solutions which fail to be main in that not all $S$ in $U$ are winning sets. These two definitions are equivalent. The solution is determined by the weights $\left(x_{i}\right)$. Let $k$ be the minimum of $\sum_{i \in S} x_{i}$ for winning sets $S$. Then $U$ is the family of all sets of weight $k$ which meet all winning sets of weight $k ; V_{U}$ is a solution if and only if no set of weight less than $k$ meets all winning sets of weight $k$. Theorem. If there exists a system $w=\left(w_{i}\right)$ of weights, all nonzero, in the strong game $G$, such that every winning set has at least half the total weight, then $G$ has at most one pseudo-main solution, which must be main simple. The theorem applies to all strong simple sixperson games. An eleven-person game shows that the hypothesis that all $w_{i}>0$ is needed. (Received February 14, 1955.)

\section{M. S. Klamkin: On a homogeneous two-dimensional low-pass filter.}

The filter considered consists of an infinite net of squares, the sides of which all contain a self-inductance $L m$ if they are parallel to the $m$-direction or a self-inductance $L n$ if they are parallel to the $n$-direction, while the vertices $(m, n)$ are connected to a perfectly conductive plate at zero potential via capacitances $C$. This filter is a generalization of the one considered by van der Pol and Bremmer in their book Operational calculus, where $L m=L n$. If one now assumes a source of voltage in parallel with the capacitor at the origin $(0,0)$, delivering a current $I_{0}(t)$ to the filter, one then has an infinite number of simultaneous partial difference equations for the voltage function at the vertices of the lattice. This voltage function is determined by use of the simultaneous operational calculus. Furthermore, by considering the special case $C=0$, and replacing the inductances $L m$ and $L n$, by resistances $R m$ and $R n$, respectively, the equivalent resistance between the points $(0,0)$ and $(m, n)$ is calculated. In particular, for $(m, n)=(1,0), R=R m / 2+(R m / \pi) \sin ^{-1}(R n-R m) /(R n+R m)$. (Received March 2, 1955.)

\section{S. G. Kneale: Axially symmetric polyharmonic functions.}

Let $\nabla_{p}^{2}$ be the $(p+2)$-dimensional Laplacian operator and let $\nabla_{p}^{2(K+1)}=\nabla_{p}^{2} \nabla_{p}^{2 \pi}$; then a solution of $\nabla_{p}^{2(K+1)} \Phi=0$ is called a polyharmonic function and, in particular, a $K$ harmonic p-function. Such functions are of particular physical interest for $K=0,1$ and $p=0,1,2,3$. Nicolesco (Les fonctions polyharmoniques, Paris, Hermann, 1936) found that harmonic functions are analytic functions satisfying Gauss's theorem of the arithmetic means and its converse. A generalized Kelvin transformation holds for polyharmonic functions and serves to define regularity at infinity. Polyharmonic functions regular in a star-shaped region are expressible as a finite sum involving harmonic functions. Finally, a uniqueness theorem holds for polyharmonic functions. 
Attention is restricted to axially symmetric harmonic and biharmonic functions. By application of known facts concerning ultra-spherical polynominals, harmonic functions are shown to possess a Laurent series analogous to the Laurent series for complex analytic functions. In terms of such a series, the author is able to discuss the isolated singularities of harmonic and biharmonic functions. The extension to other polyharmonic functions is evident. (Received March 10,1955.)

498t. K. S. Miller and L. A. Zadeh: Signal representation in terms of periodic functions.

Signal representations of the form $f(y)=\int_{0}^{\infty} K(x y) G(x) d x$, where $K(z)$ is a periodic function, are investigated. Formulas for the inverse kernel $K^{-1}(z)$ are developed in integral form and in an infinite trigonometric series. Closed forms for the integral and derivative of $K(z)$ are presented. In the case $K(z)$ is a square wave the reciprocal kernel can be written in terms of an inverse Mellin transform involving the Riemann zeta-function while the coefficients of the infinite series representation are essentially the Möbius mu function. Applications to problems in signal resolution are treated. (Received March 1, 1955.)

499. Morris Morduchow: An approximate solution, by method of averages, of a differential equation for flow in a tube with normal fluid injection at wall.

The partial differential equations for the laminar incompressible flow through a rectangular or circular tube with uniform normal velocity along the wall can be transformed into a fourth-order nonlinear ordinary differential equation with mixed boundary conditions. The equation, moreover, contains a parameter $R$ which is usually large in practice, but whose reciprocal is the coefficient of the highest derivative term. Despite the difficulties or inconveniences which might arise in obtaining numerical solutions of such an equation for a complete range of values of $R$, a simple approximate solution in terms of $R$, valid for $0 \leqq R \leqq \infty$, is obtained with relatively little difficulty by a method of averages. This solution is shown to reduce exactly to a small-perturbation solution for small $R$ and approximately to an asymptotic solution for large $R$. The solution is of particular interest in connection with transpiration or sweat-cooling of aerodynamically heated surfaces. The method of averages serves here as a comparatively simple alternative to the method of least squares. (Received March 2, 1955.)

\section{L. E. Payne: Bounds for the torsional rigidity of a composite beam.}

The cross section of an elastic beam is assumed to be composed of two or more materials possessing different elastic constants. In the St. Venant torsion problem for such a beam the stress function or the normal derivative of the warping function must satisfy certain jump conditions on the separating boundaries. It is shown in this paper that bounds can readily be obtained for the torsional rigidity of the beam. In particular an upper bound is given in terms of continuous functions which approximate the warping function, and a lower bound involves continuous functions which vanish on the outer boundary of the section and approximate the modified stress function. These bounds may be improved by the usual Rayleigh-Ritz procedure. (Received March 3, 1955.) 
501. L. A. Rubel: An estimate of the error due to modified boundary conditions in the Blasius differential equation.

In the numerical calculation of the solution $f(x)$ of the Blasius differential equation $f^{\prime \prime \prime}(x)+f(x) f^{\prime \prime}(x)=0$ under the boundary conditions of $f(0)=0, f^{\prime}(0)=0, f^{\prime}(\infty)$ $=2$, the third boundary condition is customarily modified to $f^{\prime}(M)=2$, where $M$ is some "large number." Let $f(x ; M)$ denote the solution under the modified boundary conditions. It is desirable to justify the customary use of $f(x ; M)$ as an approximation to $f(x)$. This paper provides the inequality $|f(x)-f(x ; M)| \leqq M f^{\prime \prime}(x ; M) / f(M ; M)$ for all $x$ such that $0 \leqq x \leqq M$. Since the right-hand side of the inequality contains only computable quantities, an estimate of the error may be easily computed. It is also shown that if $M>1+2(N+1)^{1 / 2}(N \geqq 3)$, then $|f(x)-f(x ; M)|<10^{-N}$ for all $x$ such that $0 \leqq x \leqq M$, so that $M=8$ yields at least 10 place accuracy, and $M=10$ yields at least 19 place accuracy. (Received February 18, 1955.)

502t. K. M. Siegel and T. B. Curtz: Inequalities involving cylindrical functions.

The Bessel functions $J_{\nu}(\nu x), J_{\nu}^{\prime}(\nu x)$ of nearly equal argument and order are bounded above by expressions which tend respectively to $J_{\nu}(\nu)$ and $J_{\nu}^{\prime}(\nu)$ as $x \rightarrow 1$. The regions in which these results improve previously known inequalities are determined. By an argument similar to that used by Watson for $J_{\nu}(\nu)$, the well-known Cauchy approximation $J_{\nu}(\nu) \sim 3^{1 / 6} \Gamma(2 / 3) / 2^{1 / 3} \pi \nu^{2 / 3}$ is shown to be an over-estimate. For the range $1 \leqq x \leqq J_{\nu}^{\prime} / \nu\left(j_{\nu}^{\prime}\right.$ the smallest positive zero of $\left.J_{\nu}^{\prime}(x)\right)$, an easily derived bound for the Neumann function $N_{\nu}(\nu x)$ is $|N(\nu x)|<1.73 J_{\nu}(\nu)$. (Received January 26, 1955.)

\section{3t. I. A. Stegun and Milton Abramowitz: The generation of} Coulomb wave functions by means of recurrence relations.

A discussion of the computation of Coulomb wave functions from their recurrence relation is given. Specifically, it is demonstrated that the regular solution $F_{L}$ and the irregular solution $G_{L}$ may be obtained recursively based on the knowledge of the functions for $L=0$. (Received January 24,1955 .)

504. H. F. Weinberger: Lower bounds for minima by finite difference methods.

Let $\lambda$ be the lowest eigenvalue of the membrane problem for the region $D$ with boundary $C$. That is, $\Delta u+\lambda u=0$ in $D, u=0$ on $C$. Let $\lambda^{h}$ be the corresponding quantity for the analogous finite difference problem $\Delta_{h} v+\lambda^{h_{v}}=0$ in $D_{h}$, where $D_{h}$ is a region consisting of entire squares of a grid with mesh size $h$, and $v$ is defined at the mesh points of $D_{h}$ and vanishes at its boundary points. Then, if $D_{h}$ contains $D$ as well as all its positive translates in the $x$ and $y$ directions of magnitudes at most $h$, we have $\lambda^{h} \leqq \lambda$. To each position of $D$ correspond certain values of $u$ at the mesh points of $D_{h}$ which are zero at the boundary points of $D_{h}$. The inequality $\lambda^{h} \leqq \lambda$ is proved by substituting these values of $u$ in the minimum principle for the finite difference problem and averaging over all admissible positions of $D$. The same result is obtained for the reciprocal of the torsional rigidity and for the lowest frequency of a clamped plate. The slightly weaker result $\nu^{h} \leqq \nu /\left(1-h \nu^{1 / 2} / 6\right)$ holds for the critical buckling load $\nu$ of a clamped plate. This research was supported by the United States Air Force through the Office of Scientific Research. (Received March 3, 1955.) 
505. C. B. Tompkins: A modified Jacobi method for diagonalizing symmetric matrices.

The Jacobi method for diagonalizing matrices reduces large off-diagonal elements to zero by means of a rotation in the space of the two coordinates involved with the element if the matrix is considered as a matrix of a quadratic form. If the off-diagonal element is $h$ and the diagonal elements of its row and column are $a$ and $b$, the rotation is $\theta$, where $\tan 2 \theta=2 h(a-b)^{-1}$. The present method is designed for use on simple computing machines or for easy coding. By considering the off-diagonal elements in order and by restricting the rotations to be from a tabulated or easily computed set the calculation is lengthened but simplified. The set of rotations might be (for example) $2^{-k}$ radians $k=1(1) n$; a single rotation is used as long as it is effective for any off-diagonal element before the next smaller rotation is used; the rotation is to be carried out (in a sense depending on the sign of $h(a-b)^{-1}$ ) if and only if the rotation considered is less than the Jacobi rotation in the formula above. Convergence to a diagonalized form is proved, and the code does not involve any divisions or roots. (Received March 3, 1955.)

\section{GEOMETRY}

506. H. S. M. Coxeter: The collineation groups of the finite planes $E G(2,3)$ and $P G(2,3)$.

The group of all collineations in the finite projective plane $P G(2,3)$ is the simple group $L F(3,3)$ of order 5616. Brahana (Ann. of Math. (2) vol. 31 (1930) p. 531) gave two matrices which generate this group, but did not attempt to find a complete set of defining relations. In terms of a different pair of generators, such relations are now found to be $S^{6}=T^{3}=(S T)^{4}=\left(S^{2} T\right)^{4}=\left(S^{3} T\right)^{3}=1, \quad\left(T S^{2} T\right)^{2} S^{2}=S^{2}\left(T S^{2} T\right)^{2}$. These are established by enumerating the 26 cosets of the subgroup $T^{3}=U^{3}=(T U)^{4}=1$, $(T U T)^{2} U=U(T U T)^{2}$ (where $U=S^{2}$ ), which is the Hessian group of order 216, i.e., the group of projective collineations leaving invariant the nine inflections of the general cubic curve in the complex projective plane. This, in turn, has a subgroup of index 9: $U^{3}=1, U V U=V U V$ (where $V=T^{-1} U T$ ), which is the binary tetrahedral group of order 24. (Received March 3, 1955.)

\section{Jesse Douglas: $A$ theorem on skew pentagons.}

Let $P_{n}, n=0,1,2,3,4$, denote the vertices of any (skew) pentagon in euclidean $m$-dimensional space, while $M_{n}$ denotes the midpoint of the side $P_{n+2} P_{n-2}$ (indices taken mod 5). The five line segments $P_{n} M_{n}$ will be called the medians of the pentagon. Theorem: (I) If $P_{n} M_{n}$ is extended to $Q_{n}$ where $M_{n} Q_{n}=\left(1 / 5^{1 / 2}\right) P_{n} M_{n}$, the five points $Q_{n}$ are the vertices of an affine-regular pentagon of convex type. (II) If $P_{n} M_{n}$ is abbreviated to $P_{n} Q_{n}^{\prime}$ where $M_{n} Q_{n}^{\prime}=\left(1 / 5^{1 / 2}\right) P_{n} M_{n}$, the five points $Q_{n}^{\prime}$ are the vertices of an affine-regular pentagon of star-shaped type. Definition of affine-regular: the parallel projection on any plane of a regular pentagon $\pi$; convex type, $\pi=\left(1, \omega, \omega^{2}\right.$, $\left.\omega^{3}, \omega^{4}\right)$ where $\omega=e^{2 \pi i / 5}$; star-shaped type, $\pi=\left(1, \omega^{2}, \omega^{4}, \omega, \omega^{3}\right)$. Cf. the author's paper Geometry of polygons in the complex plane [Journal of Mathematics and Physics (M.I.T.) vol. 19 (1940) pp. 93-130]. $\pm 1 / 5^{1 / 2}$ are the only multipliers that will produce a special pentagon of any sort, starting from an arbitrary one; otherwise the transformed pentagon may be an arbitrary one. (Received March 4, 1955.) 
508t. Frank Harary: On local balance and $N$-balance of a signed graph.

The results of this note were motivated by their applicability to certain problems in social psychology. A signed graph (s-graph) is obtained from a linear graph when some of its lines are called positive and the remaining lines negative. The sign of a cycle is the product of the signs of its lines. An $s$-graph is balanced if every cycle is positive. Two characterizations of balanced $s$-graphs were obtained in $O n$ the notion of balance of a signed graph, Michigan Math. J. vol. 2 (1953-54) pp. 143-146. An $s$-graph is locally balanced at point $P$ if every cycle through $P$ is positive. Theorem: If $G$ is balanced at $P$ and $Q$ is a nonarticulation point on a cycle containing $P$, then $G$ is balanced at $Q$. An $s$-graph is $N$-balanced if all its cycles of length $\leqq N$ are positive. A criterion for $G$ to be $N$-balanced is given and local $N$-balance is discussed. (Received February 28, 1955.)

\section{N. D. Lane: Differentiable points of arcs in conformal $n$-space.}

This paper is a generalization to $n$ dimensions of a note on differentiable points in the conformal plane, Canadian Journal of Mathematics vol. 5 (1953) pp. 512-518. Families $\tau_{r}^{(m)}$ of tangent $m$-spheres $S_{r}^{(m)}$, where $0 \leqq m \leqq n-1$ and $0 \leqq r \leqq m+1$, having contact of order $r$ with an arc at a point, are defined, and a conformal definition of differentiability, involving the tangent $(n-1)$-spheres, is given. The following properties of the families $\tau_{r}^{(n-1)}-\tau_{r+1}^{(n-1)}, r=0,1, \cdots, n-1$, and the natures of the $m$-spheres $S_{m+1}^{(m)}, m=0,1, \cdots, n-1$, lead to the classification of the differentiable points $p$ of an arc into $2^{n-1}(3 n-1)$ types. For each $r<n$, the $(n-1)$-spheres of $\tau_{r}^{(n-1)}-\tau_{r+1}^{(n-1)}$ all support, or all intersect the arc at $p$. If $S_{m}^{(m-1)}=p$, while $S_{m+1}^{(m)} \neq p$, the spheres of $\tau_{m}^{(n-1)}-\tau_{m+1}^{(n-1)}$ all support; $m=1,2, \cdots, n-1$. If $S_{n}^{(n-1)} \neq p$, it can support, intersect, or neither support nor intersect the arc at $p$ (i.e., every neighborhood of $p$ on the arc can have points in common with $\left.S_{n}^{(n-1)}\right)$. (Received February 7, 1955.)

\section{J. F. Nash: Extension of the solution of the imbedding problem to open Riemannian manifolds.}

By a simple device one can reduce the problem of (isometrically) imbedding general Riemannian manifolds to the problem for manifolds which are differentiably homeomorphic to spheres. However, this approach does not give the smallest dimensionality for the Euclidean imbedding space in general. If $n$-dimensional spheres with any arbitrary smooth Riemannian metric can always be imbedded in $N$-dimensional space, then any $n$-manifold with such a metric can be imbedded in $(n+1) N$ dimensional space. Since compact $n$-manifolds require at most $3 n^{2} / 2+11 n / 2$ dimensions, then open $n$-manifolds require at most $3 n^{3} / 2+7 n^{2}+11 n / 2$ dimensions. Possibly the work that has been done on the problem of Weyl would fit in with this device to give imbeddings of all 2-manifolds in 9-space. (Received January 31,1955.)

\section{Valdemars Punga: Indicatrix of calculus of variations and Finsler geometry.}

According to E. Cartan's point of view, Finsler geometry is envisaged as a space of line elements which is locally Euclidean. Using the concept of Caratheodory's indicatrix, Finsler space can be envisaged as point space which is locally Minkowskian. The most fundamental study of Finsler geometry and its generalizations by means of an indicatrix can be found in the papers of the Russian mathematician V. V. Vagner. 
In the present paper the author reproduces the main results of Finsler geometry using as a model 3-dimensional Euclidean space with a 2-dimensional surface-indicatrix associated with every point of the space. In this way it is possible to visualize Finsler geometry and to derive its results by the reasoning methods of synthetic geometry. (Received February 24, 1955.)

\section{2t. Moses Richardson: On finite projective games.}

Let $P G\left(k, p^{n}\right)$ be the finite $k$-dimensional projective space whose field of coordinates is the Galois field $G F\left(p^{n}\right)$. A simple game is defined by letting the players be the points of this space and letting the minimal winning coalitions be the subspaces of dimension $h$ if $k=2 h$, and of dimension $h+1$ if $k=2 h+1$. The resulting game is denoted also by $P G\left(k, p^{n}\right)$ and is termed a finite projective game. It is shown that $P G\left(2, p^{n}\right)$ is strong if $p^{n}=2$, but that there exists a blocking coalition of $2 p^{n}$ members if $p^{n}>2$, and one of $2 p^{n}-1$ members if $p^{n}>3$. If $d$ is a divisor of $n, 1 \leqq d<n$, then there exists in $P G\left(2, p^{n}\right)$ a blocking coalition of $2 p^{n}-p^{d}+1$ members. It is also shown that every $P G\left(2, p^{n}\right)$ has a main simple solution. (Received February $7,1955$. )

\section{Seymour Schuster: Pencils of polarities in projective space. I.}

A polarity $(A B C)(P p)$ in projective space of two dimensions $\left(S_{2}\right)$ is completely determined by a self-polar triangle $A B C$, and a pair of corresponding elements point $P$ and its polar line $p$. A pencil of polarities is the set of $\infty^{1}$ polarities $(A B C)(P p)$ where $A, B, C$, and $P$ are fixed while $p$ varies in a pencil of lines. Coxeter has developed this notion and has shown that two types of the classical pencils of conics are, in fact, the conics which arise from the pencils of polarities. They are the general system with four distinct points in common and the double contact system where the conics have in common two distinct points and common tangents at these points. A pentagonal pencil of polarities is defined as the $\infty^{1}$ polarities which arise from a selfpolar pentagon in which one side varies in a pencil of lines. Does this definition yield anything more general than the previous one? Investigation shows the answer to be in the affirmative in that the two triangular systems can both be derived as pentagonal pencils; and, two new systems arise to yield pencils of conics-(1) with two-point contact at a point and two other distinct points in common, and-(2) the four-point contact system. It is also shown that the remaining pencil of conics, with three-point contact cannot be derived from pentagonal pencils. (Received February 9, 1955.)

\section{4t. Seymour Schuster: Pencils of polarities in projective space. II.}

Definition: A tetrahedral pencil of polarities is the set of polarities $(A B C D)(P \pi)$ where the self polar tetrahedron $A B C D$ and point $P$ are fixed, while $\pi$, the polar plane of $P$, varies in an axial pencil. There are four tetrahedral systems $P_{4 k}^{3}$, where $k$ is $0,1,2$, or 3 , according as the axis of the pencil of planes $\pi$ meets $0,1,2,3$ edges of $A B C D$. If two polarities belong to the same: (1) $P_{41}^{3}$ system, their product is a general axial homography. (2) $P_{42}^{3}$ system, their product is a hyperbolic biaxial homography. (3) $P_{43}^{3}$ system, their product is a spatial homology. The locus of poles of a fixed plane with respect to a tetrahedral pencil is: (a) a twisted cubic passing through the vertices of the fixed self-polar tetrahedron in a $P_{40}^{3}$ pencil. (b) a conic passing through three vertices of one of the fixed self-polar tetrahedra in a $P_{41}^{3}$ pencil. (c) a line passing through two of the vertices of one of the fixed self-polar tetrahedra in a $P_{42}^{3}$ pencil. (d) a line passing through the vertex common to all the self-polar tetrahedra in a $P_{43}^{3}$ pencil. Every tetrahedral pencil yields a pencil of quadrics. Four of the thirteen non- 
degenerate pencils of quadrics can so be obtained. Each tetrahedral pencil is associated with the Segre symbol of the corresponding pencil of quadrics as follows: $P_{40}^{3}-[1111] ; P_{41}^{3}-[(11) 11] ; P_{42}^{3}-[(11)(11)] ; P_{43}^{3}-[(111) 1]$. (Received February 9, 1955.)

\section{LOGIC AND Foundations}

\section{R. S. Ledley: Computational methods in symbolic logic.}

A binary digitalization of the propositional calculus of symbolic logic has been given previously (JORSA 2:3, Aug. 1954). Based on these results, a general method for systematically generating all antecedence and consequence solutions of given form to a set of Boolean equations has been obtained. Specifically, given the set of equations $\left\{E_{s}\left(A_{1}, \cdots, A_{i}, x_{1}, \cdots, x_{k}\right)\right\}$, and a set of forms $\left\{F\left(f_{1}, \cdots, f_{j}, x_{1}, \cdots\right.\right.$, $\left.\left.x_{k}\right)\right\}$ where $E_{s}$ and $F_{s}$ are Boolean functions of the $A_{s}, x_{8}$ and $f_{s}$, and $f_{s}=f_{s}\left(A_{1}, \cdots, A_{i}\right)$ is unknown, the functions $f_{s}$ are determined such that the $\left\{F_{s}\right\}$ become antecedent solutions, i.e., $\pi_{s} F_{s} \rightarrow E_{s}$ for all $s$, or such that the $\left\{F_{s}\right\}$ become consequent solutions, i.e., $\pi_{s} E_{s} \rightarrow F_{s}$ for all $s$. The method involves the matrices $\left(E_{k \imath}\right)$ and $\left(F_{j k}\right)$ which are formed from the designation numbers of $\pi_{s} E_{s}$ and $\pi_{s} F_{s}$ respectively. The solutions are read from the matrix $\left(R_{j i}\right)$ where for antecedent solutions $\left(F_{j k}\right) \cdot\left(\bar{E}_{k i}\right)$ $=\left(\bar{R}_{i i}\right)$, and for consequent solutions $\left(\bar{F}_{j k}\right) \cdot\left(E_{k i}\right)=\left(\bar{R}_{j i}\right)$, where the matrix product involves only logical multiplication and addition. If solutions of particular forms do not exist, then the constraint $c$ under which such solutions would exist are given by $\left(V_{j}\right) \cdot\left(R_{j i}\right)=\left(C_{i}\right)$ where $\left(V_{j}\right)$ is row vector of all units, and $\left(C_{i}\right)$ is the designation number of $c$. (Received February 28, 1955.)

\section{Statistics ANd Probability}

\section{D. A. Darling (p) and Paul Erdös: A limit theorem for the maxi-} mum of normalized sums of independent random variables.

The following theorem is proved: Let $X_{1}, X_{2}, \cdots$ be independent random variables with a uniformly bounded third absolute moment, mean 0 and variance 1 . Put $S_{n}=X_{1}+X_{2}+\cdots+X_{n}$ and let $U_{n}=\max _{1 \leqq k \leqq n} S_{k} / k^{1 / 2}$. Then for $-\infty<t-\infty$, $\lim _{n \rightarrow \infty} \operatorname{Pr}\left\{U_{n}<(2 \log \log n)^{1 / 2}+2^{-1}(\log \log \log n)(2 \log \log n)^{-1 / 2}+t(2 \log \log n)^{-1 / 2}\right\}$ $=\exp \left(-\left(1 / 2 \pi^{1 / 2}\right) \exp (-t)\right)$. A similar theorem is proved for $U_{n}^{\prime}=\max _{1 \leqq k \leqq n}\left|S_{k}\right| / k^{1 / 2}$ and two strong theorems for the sequence $U_{n}$ are indicated. The method of proof consists in finding an interpolatory stochastic process for the sequence $S_{k} / k^{1 / 2}$, obtaining a limit theorem for its maximum and then an application of the invariance principle of Erdös-Kac. (Received March 3, 1955.)

517t. D. A. Darling and A. J. F. Siegert: The distribution of certain functionals of Markoff processes.

Let $S$ be an abstract space of elements $x, y, \cdots$ and $\mathcal{F}$ a Borel field of subsets of $S$; i.e. $(S, \mathcal{Z})$ is a measurable space. (i) Let $X_{n}, n=0,1, \cdots$, be a Markoff chain with values in $S$ having a transition probability $P_{j k}(x ; A)=\operatorname{Pr}\left\{X_{k} \in A \mid X_{j}=x\right\}$, where $k \geqq j, A \in \mathcal{F}$. For each $j=0,1, \cdots$ let $V(x, j)$ be a real-valued $\mathcal{F}$ measurable function such that $E\left\{\sum_{j=1}^{n}\left|V\left(X_{j}, j\right)\right| \mid X_{0}=x, X_{n} \in A\right\}<\infty$. Then the function $R_{j k}(x ; A)$ $=E\left(\exp \left\{i \xi \sum_{\nu=i}^{k} V\left(X_{\nu}, v\right)\right\} \mid X_{j}=x, X_{k} \in A\right) P_{j k}(x ; A)$ satisfies uniquely either of the integral equations $R_{j k}(x ; A)=P_{j k}(x ; A)+\sum_{\nu-j}^{k} \int_{S}\left(1-e^{-i \xi V(y, \nu)}\right) R_{j \nu}(x ; d y) P_{\nu k}(y ; A)$ or $R_{j k}(x ; A)=P_{j k}(x ; A)+\sum_{\nu=j}^{k} \int_{S}\left(1-e^{-i \xi V(y, \nu)}\right) P_{j \nu}(x ; d y) R_{\nu k}(y ; A)$. (ii) Let $X(t), t \geqq 0$, be a Markoff process with transition probability $P(x, s ; A, t)=\operatorname{Pr}\{X(t) \in A \mid X(s)=x\}$ 
and $V(x, t)$ be a real-valued $\mathcal{F}$ measurable function for each $t$ and continuous in $t$ for each $x$, and such that $V(X(t), t)$ is a measurable process with $\int_{8}^{t} E(|V(X(\tau), \tau)| \mid X(s)$ $=x, X(t) \in A) d \tau<\infty$. Then the function $R(x, s ; A, t)=E\left(\exp \left\{i \xi \int_{s}^{t} V(X(\tau), \tau) d \tau\right\}\right.$ $\mid X(s)=x, X(t) \in A) P(x, s ; A, t)$ satisfies uniquely either of the integral equations $R(x, s ; A, t)=P(x, s ; A, t)+i \xi \int_{s}^{t} \int_{S} V(y, \tau) P(x, s ; d y, \tau) R(y, \tau ; A, t) d \tau$ or $R(x, s ; A, t)$ $=P(x, s ; A, t)+i \xi \int_{s}^{t} \int_{s} V(y, \tau) R(x, s ; d y, \tau) P(y, \tau ; A, t) d \tau$. If the chain $X_{n}$ or the process $X(t)$ is temporally homogeneous and $V$ depends only on $x$, these integral equations reduce to Fredholm equations by using generating functions or Laplace transforms. When $S$ is $E_{k}$ in (ii) and the function $P$ satisfies the Fokker-Planck differential equations, $R$ satisfies two equations of the same nature. (Received March 3, 1955.)

\section{8t. Eugene Lukacs: A characterisation of the gamma distribution.}

The following theorem is proven: Let $X$ and $Y$ be two nondegenerate and positive random variables and suppose that they are independently distributed. The random variables $U=X+Y$ and $V=X / Y$ are independently distributed if, and only if, both $X$ and $Y$ have gamma distributions with the same scale parameter. The theorem is closely related to a recent result of R. G. Laha [Ann. Math. Statist. vol. 25 (1954) pp. 784-787] who characterized the gamma distribution essentially by the independence of the ratio of certain quadratic forms from the mean. However, Laha assumed that the random variables are identically distributed and that their second moments exist; both these assumptions are avoided in the theorem. (Received February 28, 1955.)

\section{TOPOLOGY}

\section{Eldon Dyer: A fixed point theorem.}

In this paper it is shown that if $M$ is the Cartesian product of finitely many compact metric chainable continua and $f$ is a continuous mapping of $M$ into itself, then some point of $M$ is its own image under $f$. Since an interval is a chainable continuum, this is a generalization of the Brouwer fixed point theorem. It is also a generalization of a theorem proved by $\mathrm{O}$. $\mathrm{H}$. Hamilton ( $A$ fixed point theorem for pseudo-arcs and certain other metric continua, Proc. Amer. Math. Soc. vol. 2 (1951) pp. 173-174). For a definition of chainable continua (called by Bing "snake-like continua") see R. H. Bing, Snake-like continua, Duke Math. J. vol. 18 (1951) pp. 653663 ; Bing also gives a number of examples of chainable continua in this paper. (Received February 22, 1955.)

\section{0t. E. R. Fadell: On spaces without isolated non cut points.}

Let $X$ denote a topological space and $f: X \rightarrow X$ a map. A point $x \in X$ is free if $f(x) \neq x . f$ is called a $\phi$-map if for every $x \in X$ there exists a free point $x^{\prime} \neq x$ which does not separate $f\left(x^{\prime}\right)$ and $x$. As a matter of convenience the identity map is allowed as a $\phi$-map. Also, let $F(f)$ denote the fixed points of $f$. If $g: X \rightarrow X$ is another map, $f$ and $g$ are said to be $F$-homotopic provided there exists a homotopy $H: X \times I \rightarrow X$ such that $H_{0}=f, H_{1}=g$, and $F\left(H_{t}\right)=F(f)$ for all $t, 0 \leqq t \leqq 1$. The following theorem holds (Bull. Amer. Math. Soc. Abstract 60-4-450): If $X$ is an ANR (compact metric) and $f: X \rightarrow X$ is a $\phi$-map, then there exists a map $g: X \rightarrow X$ which is $F$-homotopic to $f$ such that $g(X)=X$. In this note it is shown that for a Peano continuum $X$ a necessary and sufficient condition that all maps $f: X \rightarrow X$ be $\phi$-maps is that the non cut points of $X$ be dense in $X$. Also, if $X$ is a connected ANR (compact metric), a necessary and 
sufficient condition that for every map $f: X \rightarrow X$ there exist a map $g, F$-homotopic to $f$, such that $g(X)=X$ is that $X$ fail to possess an isolated non cut point. (Received February 21, 1955.)

521. Mary-Elizabeth Hamstrom: A note concerning the imbedding of upper semicontinuous collections of continua in continuous collections of continua.

Let $G$ be an upper semicontinuous collection of arcs and points in the plane such that $G$ is an arc with respect to its elements. The collection $G$ is said to be imbedded in a collection $W$ provided that each element of $G$ is contained in some element of $W$ and each element of $W$ contains one and only one element of $G$. If $n$ is a natural number and $H$ is a closed subcollection of arcs of $G$, let $H^{(n)}$ denote the collection of all elements $g$ of $H$ such that if $\epsilon$ is a positive number every open subcollection of $G$ containing $g$ contains an element of $H$ which has a subarc the distance between whose end points is less than $\epsilon$ but whose diameter is not less than $1 / n$. It is shown that if each arc $g$ of $G$ is a continuum of condensation of $G^{*}$ and is contained in a simple closed curve $J$ such that $J \cdot G^{*}=g$, and if $G^{*}-g$ has two components they lie in different complementary domains of $J$, then if $H$ is a closed subcollection of arcs of $G$, for no natural number $n$ is it true that $H^{(n)}=H$. It follows from results in the author's recent paper (Concerning the imbedding of upper semicontinuous collections of continua in continuous collections of continua, Amer. J. Math. vol. 76 (1954) pp. 793-810) that there is a continuous collection $W$ of simple closed curves in the plane such that $G$ is imbedded in $W$. (Received March 1, 1955.)

\section{2t. Erwin Kreyszig: Continuation problems in the theory of func- tions of several complex variables.}

By omitting the nonuniformizable points from the "Riemann domain" (complex $n$-dimensional analogue of the Riemann surface, see Behnke and Stein, Math. Ann. vol. 124 (1951) p. 1) one obtains a complex manifold. Continuation of complex manifolds is therefore a problem analogous to the continuation of Riemann surfaces. Let $M^{n *}$ and $M^{n}$ be complex manifolds of complex dimension $n$. Let $N^{*} \subset M^{n *}$ and $N C M^{n}$ be closed. Then if there exists a proper analytic mapping $T$ of $M^{n *}$ into $M^{n}$ which maps $M^{n *}-N^{*}$ one to one onto $M^{n}-N$, then $M^{n *}$ is called a proper analytic modification of $M^{n}$ with respect to $N$. Theorems: I. If $M^{n *}$ is a proper analytic modification of $M^{n}$ with respect to $N$, then the rings of holomorphic functions and the fields of meromorphic functions in $M^{n *}$ and $M^{n}$ are isomorphic, and the canonical isomorphism of the lattices of elements of analytic varieties in $M^{n *}-N^{*}$ and $M^{n}-N$ can be continued to a homomorphism of the lattice of elements of analytic varieties of $M^{n *}$ into that of $M^{n}$. II. If in addition to the hypotheses of I, $N^{*}$ and $N$ are irreducible varieties of the same dimension, then $T$ is one to one and maps $M^{n *}$ onto $M^{n}$; the two manifolds are therefore analytically equivalent. (Received April 6, 1955.)

\section{3t. Erwin Kreyszig: Monoidal transformations in topology.}

Monoidal transformations of algebraic geometry can be generalized and are then useful for solving problems in topology and theory of functions of several complex variables. Let $M^{n}$ be a complex manifold of complex dimension $n$ and $N C M^{n}$ an irreducible analytic variety of complex dimension $k$. Then $N$ can be replaced by a fibre bundle $N^{*}$ with the base $N$ and a complex $n-k-1$-dimensional projective space 
as fibre. Let us call this a $\sigma^{n, k}$-process; it is a generalization of Hopf's $\sigma$-process (Rend. Mat. Roma vol. 10 (1951) p. 169) and is independent of the choice of the local coordinates in $M^{n} . M^{n *}=\left(M^{n}-N\right) \cup N^{*}$ is again a complex manifold. Every projective space $S^{a_{1}} \times S^{a_{2}} \times \cdots \times S^{a_{m}}$ (where the index $a_{\mu}$ indicates the complex dimension) can be obtained from another (of the same complex dimension) by using certain $\sigma^{n, k}$-processes. Because $\sigma^{n, k}$-processes are special modifications of complex manifolds, all theorems of the previous note can be applied to these processes for example in order to investigate the behavior of holomorphic and meromorphic functions with respect to different closures of the complex affine space. (Received April 6, 1955.)

\section{W. T. Kyner: A generalization of the Borsuk and Borsuk- Ulam theorems.}

Let $E$ be a real, infinite-dimensional, linear topological space with a bounded convex neighborhood of the origin. Let $V$ be a closed convex body, symmetric with respect to the origin and let $S$ be its boundary. A map $f$ of $S$ into $E$ is said to be antipodal if $f(x)=-f(-x)$ for all $x$ in $S$. If $F$ is a compact map, the map $f(x)=x-F(x)$ is said to be a compact displacement of $S$. Using Rothe's theory of topological order, the author has obtained the following Borsuk type theorem: The order of the origin with respect to a nonzero, antipodal, compact displacement of the set $S$ is odd. This implies that if $F$ is a compact map defined on $V$, and if $F$ is antipodal on $S$ (or homotopic to an antipodal map), then $F$ has a fixed point in $V$. If one adds the additional restriction that $F(S)$ be closed, one obtains a generalization of the Borsuk-Ulam theorem: If $F$ is a strongly compact, antipodal map of $S$ into $E$, then there is at least one point in $S$ that maps onto the origin of $E$. (Received March 3,1955.)

\section{A. B. Novikoff: The intersection of convex sets.}

Let $C$ be a convex set in $E_{n}$ containing the origin in its exterior, and let $\lambda C$ denote its homothetic enlargement from the origin in the ratio $\lambda: 1$. In a paper on conformal mapping, M. Shiffman used the lemma that for $n=2$ the intersection of the boundaries of $C$ and $\lambda C$ consists of at most 2 connected components. He conjectured that for $n \geqq 3$, the intersection consists of at most one connected component. This is established. Under certain restrictions on the topology, the same result holds for topological vector spaces in general. (Received January 24, 1955.)

\section{6t. C. D. Papakyriakopoulos: On the ends of knot groups.}

The main result is the following: If $K$ is a polygonal knot in the 3-dimensional sphere $S$ such that $S-K$ is aspherical, then its group $\pi_{1}(S-K)$ has two ends or one end according as $\pi_{1}(S-K)$ is free cyclic or not. This clarifies a result of E. Specker (Die erste Cohomologiegruppe von Ueberlagerungen, Comment. Math. Helv. vol. 23 (1949), see p. 329). The main result is a consequence of the following two theorems: (1) Let $M$ be an aspherical finite 3-dimensional manifold whose fundamental group $\pi_{1}(M)$ is infinite. Suppose that the injection $\pi_{1}\left(N_{i}\right) \rightarrow \pi_{1}(M)$ is an isomorphism for each component $N_{i}$ of the boundary $N$. Then $\pi_{1}(M)$ has one end. (2) Let $M$ be an aspherical 3-dimensional manifold whose boundary $N$ is an orientable surface of genus one, and suppose that the injection $\pi_{1}(N) \rightarrow \pi_{1}(M)$ is not an isomorphism. Then $M$ is finite and orientable, and $\pi_{1}(M)$ is free cyclic. So $\pi_{1}(M)$ has two ends. (Received February 7, 1955.) 
527t. R. L. Plunkett: A fixed point theorem for continuous multivalued transformations.

Using one definition of continuity for multi-valued transformations (see Strother, Proc. Amer. Math. Soc. vol. 4 (1953) pp. 988-993), it is proved in this paper that a nondegenerate continuous curve (compact, locally connected, metric, continuum) has the fixed point property for continuous multi-valued transformations if and only if it is a dendrite. This is an extension of a theorem in the above paper which states that a bounded closed interval of real numbers has the fixed point property. A corollary to the theorem proved in this paper is: If $X$ and $Y$ are nondegenerate continuous curves, then $X \times Y$ does not have the fixed point property for continuous multi-valued transformations. (Received February 28, 1955.)

\title{
528t. A. H. Stone: Metrisability of decomposition spaces.
}

Let $f$ be a continuous mapping of a metric space $S$ onto a topological space $E$. If $f$ is closed, the following statements are equivalent: (1) $E$ satisfies the first countability axiom, (2) for each $p \in E$, the frontier of $f^{-1}(p)$ (in $S$ ) is compact, (3) $E$ is metrisable. (This completes results of Vainstein, Whyburn, and Hanai.) Sufficient conditions are given for $E$ to be metrisable when $f$ is open, or when $f$ is quasi-compact and $S$ is locally compact. A simple characterization is obtained of the case in which $f$ is simultaneously open and closed; in this case $E$ is always metrisable. (Received February 23,1955 .)

\section{Fred Supnick: On a class of 3-manifolds and the bioduplication} problem.

Bioduplicants (e.g. proteins, nucleic acids, nucleoproteins) are here represented by 3-manifolds $K\left(c_{1}, \cdots, c_{n}\right)$ partitioned into 3-cells $c_{i}$ (the building blocks) where $c_{i}$ and $c_{j}(i \neq j)$ either have no points in common, or have a finite number of disjoint 2-cells in common (the bonding loci). The $c_{i}$ and their junction "caps" are presumed to be finite-polyhedral. Let $F$ and $G$ be noninterlinking entities resulting from a bioduplication process; it is presumed that neither ruptures during separation. This is formulated topologically as follows: Let $F\left(f_{1}, \cdots, f_{n}\right)$ and $G\left(g_{1}, \cdots, g_{n}\right)$ be partitioned 3-manifolds with $I(F) \cdot I(G)=0$ which may be isotopically deformed without interiors overlapping to $F^{\prime}, G^{\prime}$ and $F^{\prime \prime}, G^{\prime \prime}$ such that (i) $\left(F^{\prime}+G^{\prime}\right)$ is a partitioned 3manifold whose 3 -cells are $\left(f_{i}^{\prime}+g_{i}^{\prime}\right)(i=1, \cdots, n),\left(F^{\prime}+G^{\prime}\right)$ being isotopically deformable to $F$ and to $G$ so that $\left(f_{i}^{\prime}+g_{i}^{\prime}\right)$ coincides with $f_{i}$ and $g_{i}$ respectively, and (ii) $F^{\prime \prime}$ and $G^{\prime \prime}$ fall on opposite sides of a surface topologically equivalent to a plane. It is shown that it is possible to isotopically deform $F$ to $K$ so that any line perpendicular to a plane $P$ and intersecting $K$, intersects it only in a line segment all of whose points belong to the same 3-cells. That is, $F$ has a 2-dimensional structure. (Received March 2, 1955.)

\author{
R. D. SCHAFER, \\ Associate Secretary \\ L. A. MACCoLL, \\ Editor, Proceedings of the Symposium
}

